This item was submitted to Loughborough's Institutional Repository (https://dspace.lboro.ac.uk/) by the author and is made available under the following Creative Commons Licence conditions.

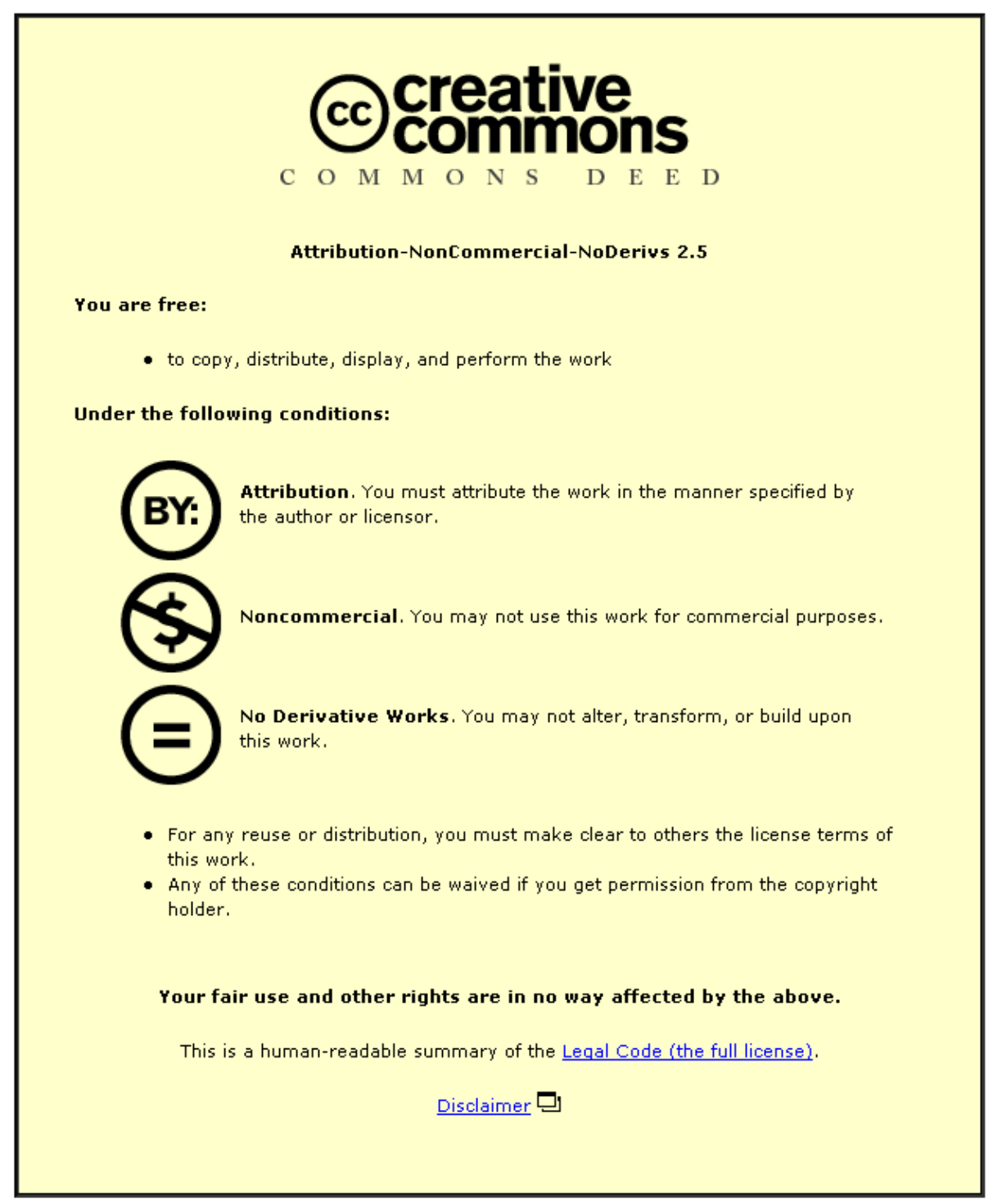

For the full text of this licence, please go to: http://creativecommons.org/licenses/by-nc-nd/2.5/ 


\title{
Computational Study of Aero-acoustic Sources in Perforate Silencers
}

\author{
C. D. Pokora* and G. J. Page ${ }^{\dagger}$ \\ Loughborough University, Leicestershire, United Kingdom.
}

\begin{abstract}
Reynolds Averaged Navier Stokes and Large Eddy Simulations of two perforate plates at a overall pressure ratio of 1.45 have been performed to allow analysis of the sensitivity of acoustic noise sources to porosity. Two geometries are presented: A $23 \%$ porosity and a $40 \%$ porosity $1 \mathrm{~mm}$ plate with $2 \mathrm{~mm}$ diameter holes. Results presented in this paper show the initial jetlet and fully merged jet flow-field to be sensitive to the porosity and the presence of partial holes around the circumference of the plate. The increase in porosity reduces the available entrainment flow, and increases the local jetlet interaction and resultant turbulence levels. This interaction fundamentally changes the flow structure from coherent vortex rings (found at low porosity) to a helical structure. The $2^{\text {nd }}$ and $4^{\text {th }}$ order spatiotemporal correlation $R_{i j}$ and $R_{i j, k l}$ are presented as suggested validation data for acoustic source modeling together with far-field noise spectra obtained via a Ffowcs-Williams \& Hawkings surface integral method.
\end{abstract}

\section{Introduction}

Orifice plates and fluid throttle screens are common devices in climate control systems of aircraft, ${ }^{1}$ as well as in process plant piping systems, ${ }^{2}$ and can be used as pulsation suppression devices for reciprocating compressor and pressure reducing devices for gas services. It is known that the restriction of orifices can cause high level tonal noise in certain conditions and induce severe vibrations under high pressure ratio. The mechanisms and scaling laws for noise radiated from subsonic airflow through single and multiholed orifice plates have been studied experimentally. ${ }^{3}$ Such geometries can be categorised as dissipative flow silencers and are used to control noise within duct type flows due to their broadband attenuation characteristics. An example of this can be seen in the design of car silencers. ${ }^{4}$ Work by Itsuro et $\mathrm{al}^{2}$ showed that the noise level for a multi perforated plate is lower than that of a single hole orifice in high pressure ratio conditions, whilst the flow rate of a multi perforated plate is larger than that of a single hole orifice.

Within this study, the mentality is to view the perforated plate as multiple orifices, each producing a separate jet ('jetlet'). It is therefore pertinent to consider single jet knowledge and aero-acoustic relations. Single jet noise modeling techniques have previously been developed which use a Reynolds Averaged CFD solution to provide turbulence statistics as input to a Lighthill or Lilley approach to compute far-field noise. Whilst these methods are generally successful in the $90^{\circ}$ sideine direction, prediction in the dominant downstream arc are often poor. ${ }^{5,6}$ Large Eddy Simulation (LES) is able to predict spatio-temporal noise sources within the aerodynamic domain whilst a suitable noise propagation techique should provide accurate far-field noise predictions for all angles. Tyically the predicted pressure fluctuations are propagated to the far-field using a Kirchoff or Ffowcs-Williams \& Hawkings (FWH) surface integral method. In addition in LES, a subgrid-scale (sgs) model is used to account for the unresolved scales of motion in the flow. On a sufficiently fine grid, the subgrid-scale model has no significant effect on the evolution of the shear layer flow. ${ }^{7}$ However for high Reynolds number flows such a high-fidelity simulation is not currently feasible and it has been shown that modications to the sgs to reduce the filter width results in significant changes to the evolution of the initial flow-field. ${ }^{8}$ Initialisation of a flow simulation at the inlet to the domain requires the specification of an inlet condition that provides the correct representation of the fundamental characteristic of the flows. In

${ }^{*}$ Research Associate, Dept. Aeronautical and Automotive Eng., AIAA Member

${ }^{\dagger}$ Senior Lecturer, Dept. Aeronautical and Automotive Eng., AIAA Member. 
typical noise studies (specifically jet noise), past research has mainly used either a uniform inlet condition ${ }^{9}$ or a hyperbolic tangent function to initialise the flow. ${ }^{10}$ In both cases noise was superimposed onto the inlet condition in order to promote the onset of turbulence in the flow. The current noise study does not suffer from any of these problems as the inlet geometry (straight pipe) and short nozzle length (perforate plate thickness) generates the correct turbulence and boundary layer development itself, removing the requirement to specify an inlet condition with accurate turbulent statistics.

The aim of this work is to perform computational simulations to provide an improved understanding of the fluid mechanics and potential aero-acoustic sources within multiple perforate geometries. This requires the collection of fundamental data on the performance of perforates, the acoustic sources, and attenuation characteristics. It should be noted that the flow and noise within perforate environment designs is extremely complex and highly geometry specific and as a consequence the test programme has been restricted to a fundamental flow study using both steady RANS and unsteady LES. This allows the objectives of obtaining basic flow understanding from the RANS and unsteady statistics and acoustic sources from the LES to be achieved. The fundamental property of jet turbulence that acts as the acoustic source in broadband jet mixing noise (Lighthill ${ }^{11,12}$ ) is the $4^{\text {th }}$ order two-point two-time fluctuating velocity correlation $\left(\mathrm{R}_{i j, k l}\right)$ and will be presented as part of this work.

\section{Methodology}

Meshes employed within this study are unstructured and mixed element and use a compressible, densitybased Reynolds Averaged Navier-Stokes solver. The unstructured nature allows great flexibility in grid generation in order to handle complex geometry of industrial interest. The drawback is that, compared to a block-structured methodology, there is an overhead in computer memory and run-time, and unstructured spatial discretisation schemes are difficult to extend to more than second order accuracy.

The discretisation has been improved so as to avoid excessive dissipation of resolved eddies, and subgrid scale models incorporated. The important features are summarised below.

\section{A. Governing Equations}

Employing Cartesian tensor notation and the conservative variables $\left(\rho, \rho u_{i}, E\right)$, the governing time dependent equations in terms of spatially filtered, Favre-averaged compressible N-S equations can be expressed as

$$
\frac{\partial}{\partial t} \int_{\Gamma} Q d V+\int_{\partial \Gamma} F(Q) \cdot n d S+\int_{\partial \Gamma} G(Q) \cdot n d S=0,
$$

where,

$$
Q=\left[\begin{array}{c}
\bar{\rho} \\
\bar{\rho} \widetilde{u} \\
\bar{\rho} \widetilde{v} \\
\bar{\rho} \widetilde{w} \\
\widetilde{E}
\end{array}\right], F(Q) \cdot n=\left[\begin{array}{c}
\bar{\rho} \widetilde{U}_{n} \\
\bar{\rho} \widetilde{U}_{n} \widetilde{u}+\hat{n}_{x} \bar{p} \\
\bar{\rho} \widetilde{U}_{n} \widetilde{v}+\hat{n}_{y} \bar{p} \\
\bar{\rho} \widetilde{U}_{n} \widetilde{w}+\hat{n}_{z} \bar{p} \\
\widetilde{U}_{n}(\widetilde{E}+\bar{p})
\end{array}\right],
$$

and $G(Q)$ contains viscous and conduction flux terms. The finite volume discretization provides an implicit filter for the large eddies and - denotes unweighted filtered variables and $\sim$ density weighted filtered variables. The spatial filter size is computed at every node from the control volume surrounding the node. The finite volumes are created from the median-dual of the original unstructured mesh which may contain tetrahedra, hexahedra, pyramids and prisms.

\section{B. Discretisation}

The fluxes through the median dual control volume faces are accumulated by looping over all the edges connecting the nodes. For an edge $i j$ that connects nodes $i$ and $j$, the flux is computed using a second-order accurate scheme of Moinier ${ }^{13}$ 


$$
F_{i j}=\frac{1}{2}\left[F\left(Q_{i}\right)+F\left(Q_{j}\right)-\text { smoothing }\right],
$$

The smoothing term is defined as

$$
\text { smoothing }=\left|A_{i j}\right| \varepsilon\left(L_{j}^{l p}(Q)-L_{i}^{l p}(Q)\right),
$$

where $L^{l p}$ is the pseudo-Laplacian and

$$
\left|A_{i j}\right|=\partial F / \partial Q
$$

For LES it is essential that the smoothing term should be kept as small as possible so as to avoid unphysical dissipation of the resolved eddies. Temporal discretisation uses a standard third order accurate, three-stage Runge-Kutta algorithm.

\section{The $k-\epsilon$ model}

The Reynolds-averaged Navier-Stokes (RANS) equations require the turbulent Reynolds stresses $\rho \overline{u_{i}^{\prime} u_{j}^{\prime}}$ to be modelled. In the current work turbulence closure is achieved using the standard $k-\epsilon$ model, where turbulent viscosity is given by

$$
\mu_{t}=\rho C_{\mu} \frac{k^{2}}{\epsilon}
$$

which requires two additional modelled transport equations to be solved for turbulent kinetic energy and dissipation rate are:

$$
\begin{gathered}
\frac{\partial(\rho k)}{\partial t}+\frac{\partial}{\partial x_{j}}\left(\rho u_{j} k\right)=\frac{\partial}{\partial x_{j}}\left(\left(\mu+\frac{\mu_{t}}{\sigma_{k}}\right) \frac{\partial k}{\partial x_{j}}\right)+P_{k}-\rho \epsilon \\
\frac{\partial(\rho \epsilon)}{\partial t}+\frac{\partial}{\partial x_{j}}\left(\rho u_{j} \epsilon\right)=\frac{\partial}{\partial x_{j}}\left(\left(\mu+\frac{\mu_{t}}{\sigma_{\epsilon}}\right) \frac{\partial \epsilon}{\partial x_{j}}\right)+\frac{\epsilon}{k}\left(C_{\epsilon 1} P_{k}-C_{\epsilon 2} \rho \epsilon\right)
\end{gathered}
$$

where the production of turbulence kinetic energy $\left(P_{k}\right)$ is given by:

$$
P_{k}=-\rho \overline{u_{i}^{\prime} u_{j}^{\prime}}\left(\frac{\partial \overline{u_{i}}}{\partial x_{j}}\right)
$$

\section{Sub Grid Scale Model}

The standard Smagorinsky SGS model ${ }^{14}$ defines the subgrid scale viscosity as

$$
\mu_{\text {sgs }}=\rho l_{\text {smag }}^{2} \sqrt{2 S_{i j} S_{i j}},
$$

where the Smagorinsky length scale is,

$$
l_{\text {smag }}=C_{s} \Delta
$$

the strain rate is,

$$
S_{i j}=\frac{1}{2}\left(\frac{\partial u_{\mathrm{i}}}{\partial x_{\mathrm{j}}}+\frac{\partial u_{\mathrm{j}}}{\partial x_{\mathrm{i}}}\right)
$$

the filter width $\Delta$ is determined from the cube root of the control volume and $C_{s}$ is a model constant.

A typical hexahedral mesh used for engineering calculations resolves the gradients in the boundary layer by decreasing spacing normal to the wall. This results in moderate to high aspect ratios close to the wall. The consequence is that the filter width stays relatively large and excessively high values of $\mu_{\text {sgs }}$ are found close to the wall. A 'purist' approach to LES would suggest that element aspect ratios should be significantly reduced to be closer to unity - but in practical terms the large increase in number of elements would render the calculation impractical. 
In the inner region of the Baldwin-Lomax or Cebeci-Smith mixing length RANS model, the turbulent viscosity takes a similar form to the sub-grid scale viscosity of the Smagorinsky model

$$
\left(\mu_{t}\right)_{i}=\bar{\rho} l_{b l}^{2}|\bar{\omega}|
$$

where the mixing length is in this case

$$
l_{b l}=\kappa y\left[1-\exp \left(\frac{y^{+}}{A^{+}}\right)\right]
$$

Since the Smagorinsky SGS model only accounts for the modelled part of the total stress, this should be smaller than the Reynolds stress predicted by the mixing length model. Hence, we have a way of restricting the excessive length scale in the Smagorinsky model,

$$
l_{\text {smag }}=\min \left(C_{s} \triangle, \kappa y\right)
$$

This can be interpreted as using a RANS model near the wall, and is similar to the Detached Eddy Simulation (DES) approach in that we are defining a length scale as the minimum of the RANS model length scale and a SGS length scale based upon the local element size. Unlike DES, which aims to use the RANS model for the whole of the boundary layer, the objective here is to use the RANS model only in the inner region of the boundary layer in order to improve the prediction of the wall shear stress.

\section{E. Second- and Fourth-Order Correlations}

The usual Reynolds decomposition is adopted to separate time-mean (statistically stationary) and fluctuating components of the instantaneous velocity field:

$$
u_{i}^{\prime}(\vec{x}, t)=u_{i}(\vec{x}, t)-U_{i}(\vec{x})
$$

Single point Reynolds stresses are defined as usual, e.g. for the rms of the axial turbulent normal stress

$$
u_{1}^{r m s}(\vec{x}, t)=\sqrt{\overline{u_{1}^{\prime}(\vec{x}, t)^{2}}}
$$

In addition, for spatio-temporal characteristics of the turbulence, the definitions adopted for the normalised $2^{\text {nd }}$ and $4^{\text {th }}$ order two-point, two-time correlations are as follows, let A,B,C identify particular spatial / temporal co-ordinate pairs:

Then:

$$
\begin{aligned}
& A=(\vec{x}, t) \\
& B=(\vec{x}+\vec{\eta}, t) \\
& C=(\vec{x}+\vec{\eta}, t+\tau)
\end{aligned}
$$

$$
\begin{gathered}
R_{i j}(\vec{x}, \vec{\eta}, \tau)=\frac{\overline{u_{i}^{\prime}(A) u_{j}^{\prime}(C)}}{\sqrt{\overline{u_{i}^{\prime}(A)^{2}}} \sqrt{\overline{u_{j}^{\prime}(B)^{2}}}} \\
R_{i j, k l}(\vec{x}, \vec{\eta}, \tau)=\frac{\overline{u_{i}^{\prime}(A) u_{j}^{\prime}(A) u_{k}^{\prime}(C) u_{l}^{\prime}(C)}-\left(\overline{u_{i}^{\prime}(A) u_{j}^{\prime}(A)}\right)\left(\overline{u_{k}^{\prime}(B) u_{l}^{\prime}(B)}\right)}{\left(\overline{u_{i}^{\prime}(A)^{4}}\right)^{\frac{1}{4}}\left(\overline{u_{j}^{\prime}(A)^{4}}\right)^{\frac{1}{4}}\left(\overline{u_{k}^{\prime}(B)^{4}}\right)^{\frac{1}{4}}\left(\overline{u_{l}^{\prime}(B)^{4}}\right)^{\frac{1}{4}}-\sqrt{\overline{u_{i}^{\prime}(A)^{2}}} \sqrt{\overline{u_{j}^{\prime}(A)^{2}}} \sqrt{\overline{u_{k}^{\prime}(B)^{2}}} \sqrt{\overline{u_{l}^{\prime}(B)^{2}}}}
\end{gathered}
$$

Simpler correlations of interest may be obtained by selecting specific values of $\vec{\eta}$ and $\tau$. For example, the auto-correlation function is obtained by setting $\vec{\eta}=0$ nd the spatial correlation function is obtained by setting $\tau=0$.

Integral length scales may be extracted from these correlations, e.g at $2^{\text {nd }}$ order level :-

$$
L_{i j}^{k}(\vec{x})=\int_{0}^{\infty} R_{i j}\left(\vec{x}, \eta_{k}, 0\right) d \eta_{k}
$$

where $\mathrm{k}$ indicates the component of the separation vector along which the integration is carried out. For example, the streamwise integral scale is evaluated as $L_{11}^{1}$ and the transverse integral scale as $L_{11}^{2}$. Note that in practice these integral scales are evaluated up to the first zero crossing of the correlation function (as in Fleury et $\left.\mathrm{al}^{15}\right)$. 


\section{Computational Grids}

Two distinct test configurations have been designed to investigate and characterise the main fluid mechanics and aeroacoustic mechanisms associated with perforate noise.

The first test configuration includes two geometries with varying porosity perforates mounted at the end of a $47.5 \mathrm{~mm}$ internal diameter pipe. The two perforates have open areas of $23 \%$ and $40 \%$ with $2 \mathrm{~mm}$ diameter holes $\left(D_{j}\right)$ resulting in $\approx 130$ and $\approx 226$ holes respectively. These models have designations of [R23-2] and [R40-2] and have been used for RANS calculations to investigate the basic jet flowfield (jet mixing and shock associated) from isolated perforates.

The second test configuration uses the same porosity perforates but with a reduced plate diameter of $21 \mathrm{~mm}\left(D_{p}\right)$. This reduces the number of holes to $\approx 25$ and $\approx 44$ for the $23 \%$ and $40 \%$ porosity perforates respectively. The reduced number of holes reduces the computational cost therefore allowing for LES calculations to be performed. These models have designations of [L23-2] and [L40-2] and have been conducted for a single overall pressure ratio of 1.45 resulting in pipe Reynolds number $R e_{\text {pipe }} \approx 97,000$, jetlet Reynolds number $R e_{\text {jetlet }} \approx 32,000$ and equivalent open area jet Reynolds number $\operatorname{Re}_{\text {jet }(L 23-2)} \approx 77,000$ and $\operatorname{Re}_{\text {jet }(L 40-2)} \approx 102,000$.

The commercial grid generator 'Centaur' was used in this study to mesh the geometry illustrated in Figure 1a-c. The hole pattern and spacing for the two porosities can be seen in Figure 1b and c. The presence of partial holes around the circumference can be identified and their potential to effect the flow development noted. Figure 2a-d illustrates the LES grid for the L40-2 geometry for the XZ plane at Y=0mm and the YZ plane $1 \mathrm{~mm}$ downstream of the perforate plate exit. Prism layers were used adjacent to the walls of the orifices, with tetrahedral elements elsewhere. The tetrahedra were increased in size away from the areas of interest. Source terms were used to control grid spacing where the jetlet shear layers were expected (Figure $2 \mathrm{~b}$ ) and the overall region of the merged jet plume (Figure 2a). As the CFD solver uses a medium dual approach, the number of unknowns is equal to the number of nodes in the mesh.

Grid resolution is critical to obtaining a high level of resolved turbulence reducing the errors incurred through large SGS model contributions. A good indicator of the quality of the computational mesh is the ratio of mean sub grid scale modelled viscosity to laminar viscosity $\mu_{t} / \mu$. This has been used to aid mesh refinement. Table 1 shows the final mesh sizes for the test configurations and perforate porosity. Note, each hole resolution is identical between perforate porosities (144,000 nodes per hole). Figure 3 shows contour plots of this ratio for the final mesh on the $\mathrm{XZ}$ plane at $\mathrm{Y}=0 \mathrm{~mm}$. This provides evidence that the grid refinement (prism layers and node clustering) has increased the resolved turbulence around the holes and downstream until $x / D_{j}=2$ producing values of between 1-3. The grid stretching employed further downstream can be seen to match the growth of the turbulent structures helping to maintain a ratio value $<$ 3 until $x / D_{j}=10$. This covers the main area of interest for this study. Further downstream the mesh becomes coarser to reduce computational cost, and as a consequence the viscosity ratio increase as axial distance increases to a peak value of 35 .

\begin{tabular}{||c|c|c|c|c||}
\hline \hline Solver & Definition & Porosity & Node count & Element count \\
\hline \hline RANS & R23-2 & $23 \%$ & 105.45 Million & 283.39 Million \\
RANS & R40-2 & $40 \%$ & 106.04 Million & 286.32 Million \\
\hline \hline LES & L23-2 & $23 \%$ & 34.88 Million & 126.37 Million \\
LES & L40-2 & $40 \%$ & 37.37 Million & 140.59 Million \\
\hline \hline
\end{tabular}

Table 1: RANS test configuration grid sizes 


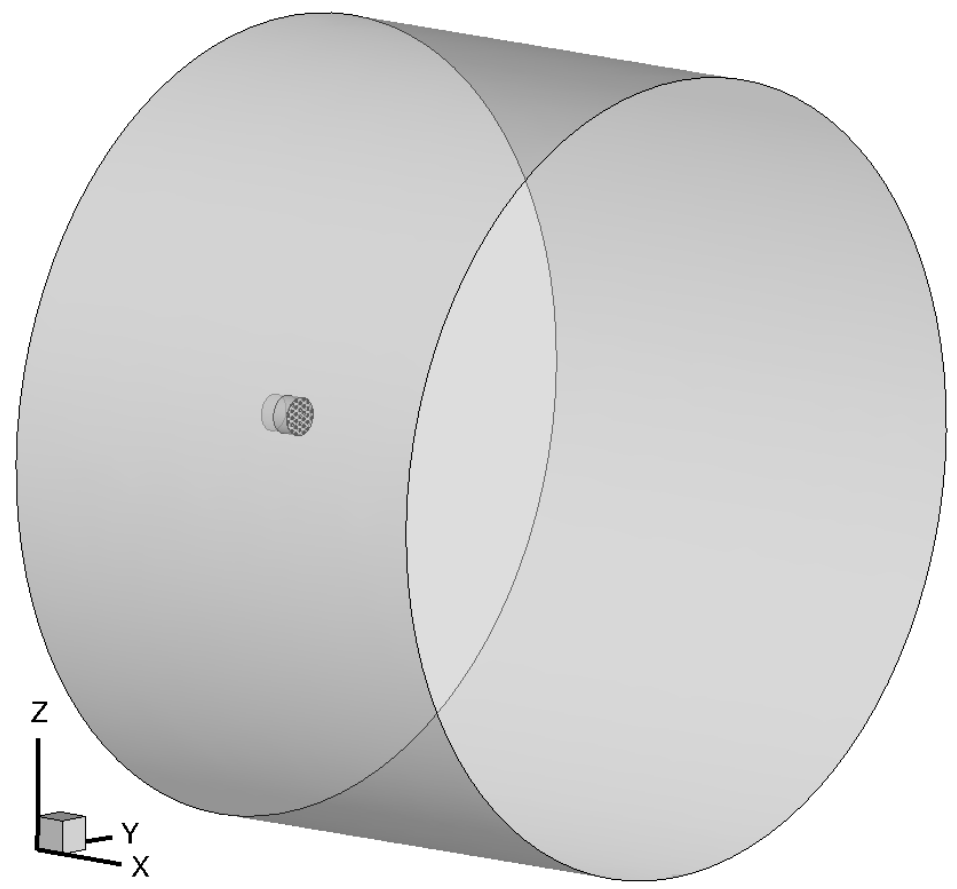

(a) XZ Plane

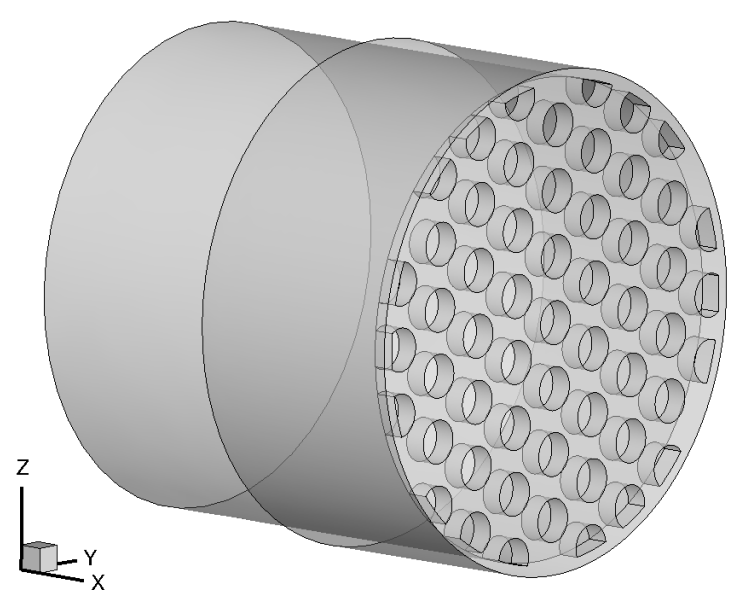

(b) XZ Plane (detailed single hole)

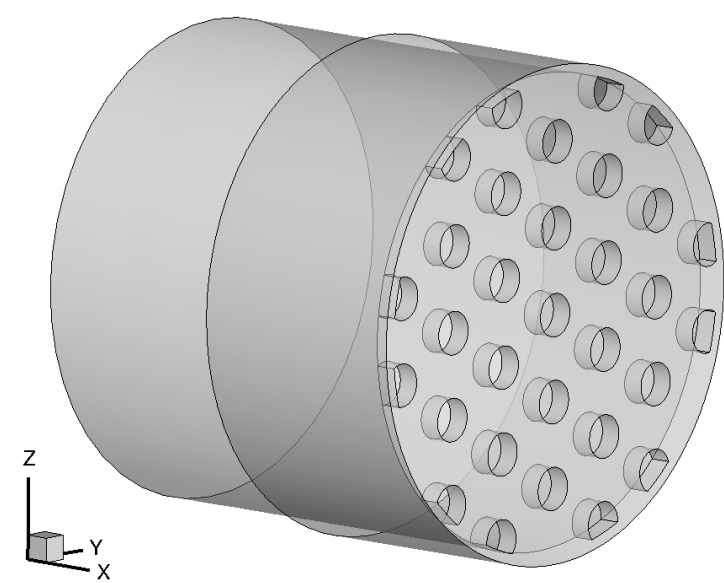

(c) ZY Plane

Figure 1: Illustrations of the $23 \%$ and $40 \%$ geometry for the LES simulations (RANS geometries are comparable) 


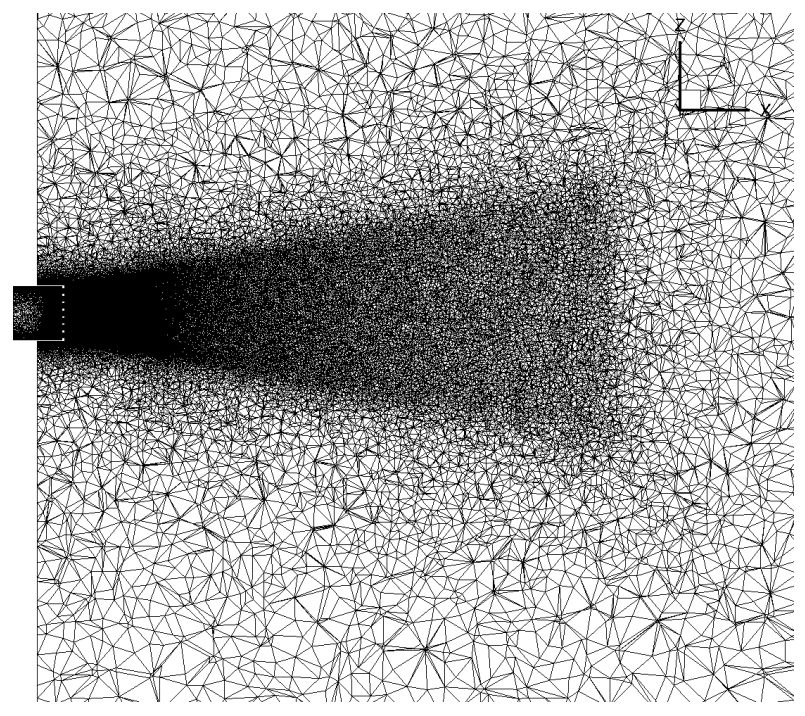

(a) XZ Plane

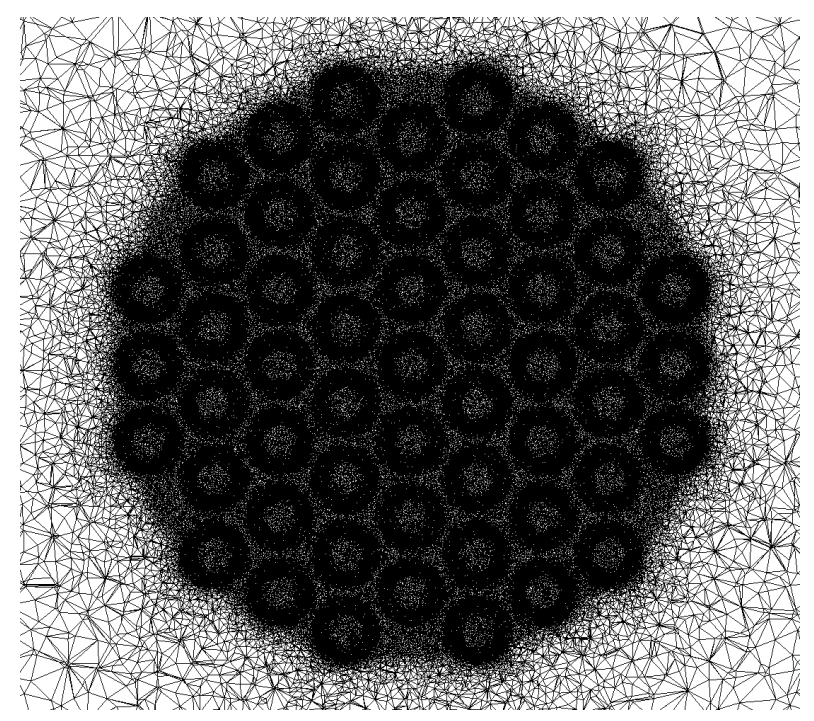

(c) ZY Plane

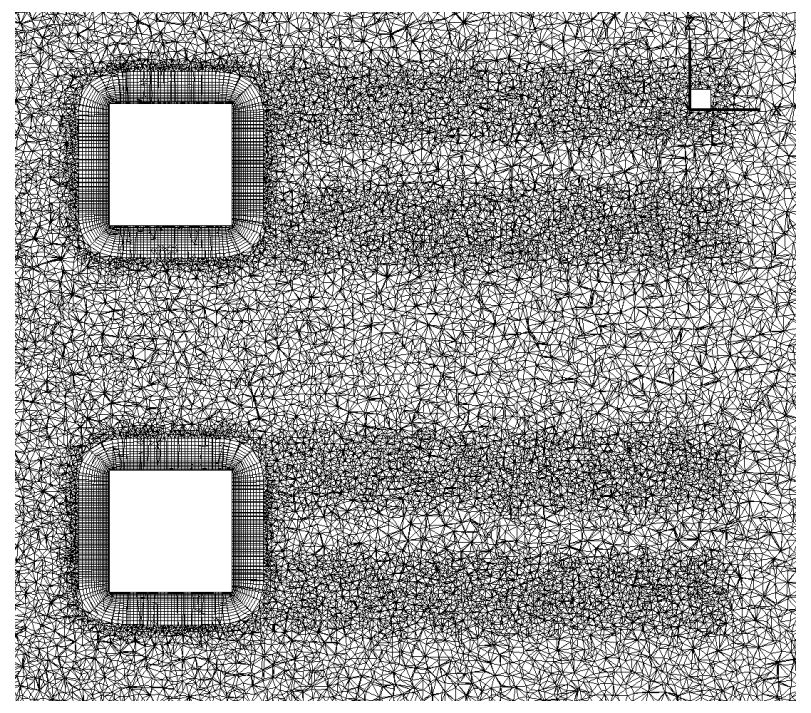

(b) XZ Plane (detailed single hole)

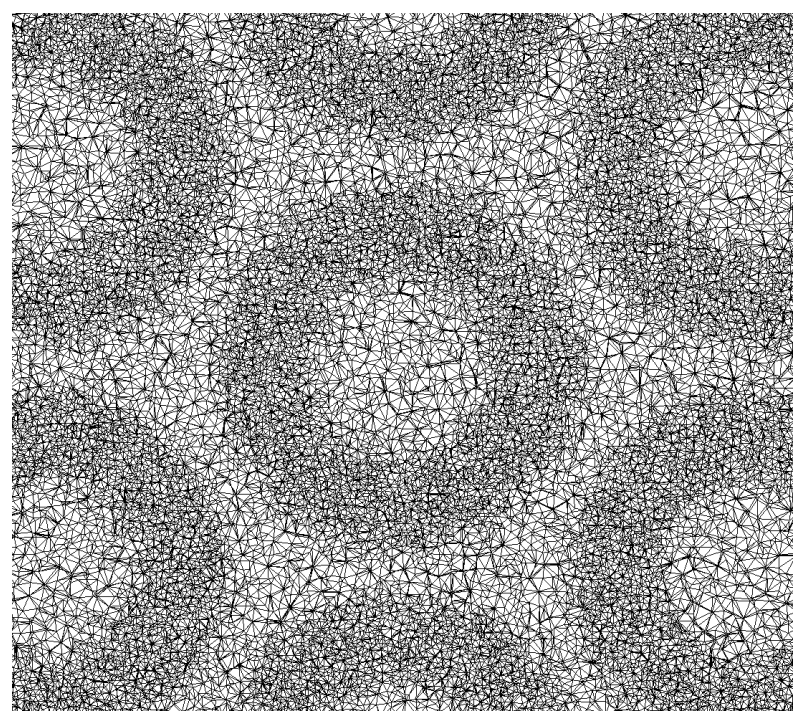

(d) ZY Plane (detailed single hole)

Figure 2: Illustrations of LES L40-2 grid (L23-2 comparable) 


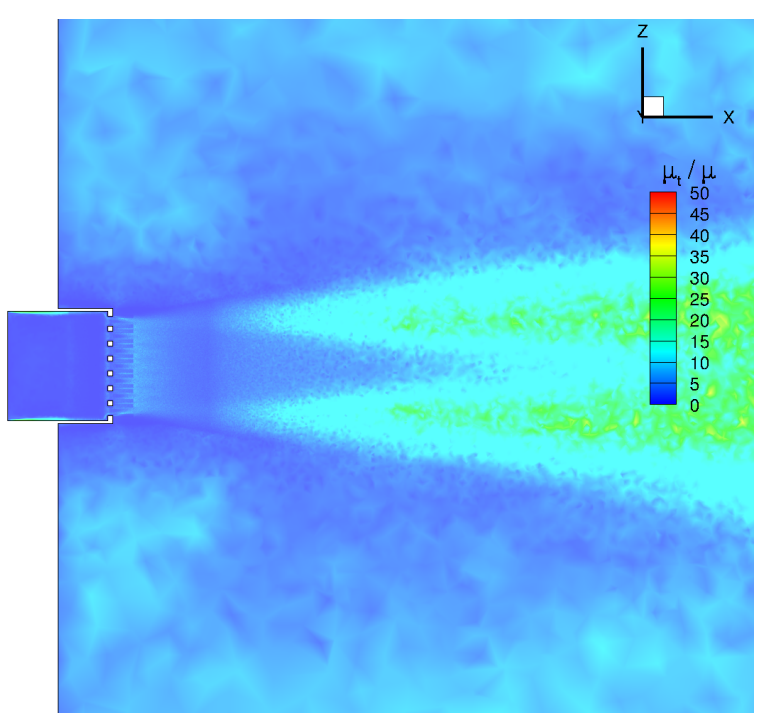

(a) XZ Plane

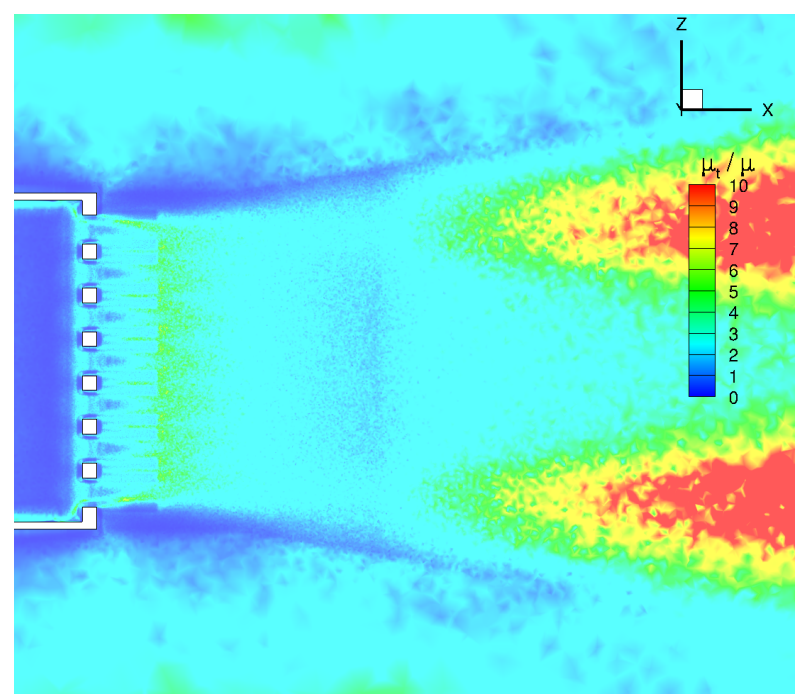

(b) XZ Plane (detailed perforate plate region)

Figure 3: Ratio of SGS to laminar viscosity $\mu_{t} / \mu$ at $\mathrm{Y}=0 \mathrm{~mm}$

\section{Results}

\section{A. Flow Statistics}

The flow simulated on each geometry is performed with a single nominal pressure ratio of 1.45 for both RANS and LES calculations. Of particular interest in the simulations is the effect of perforate porosity for a given inflow pipe diameter $\left(D_{p}\right)$. This inevitably reduces the number of holes present within the low porosity cases, and changes the number and influence of the partial holes on the outer circumference of the plate. This will be assessed in the following section.

Results from the RANS simulations for both R23-2 and R40-2 geometries are illustrated in Figure 4a-f and 5a-f in the form of XY and YZ planes at various axial locations of axial velocity and tke contours. Figure 4a-f provides evidence that the porosity of the plate changes the potential core lengths of the jetlets from each of the holes. With the increase in porosity comes a reduction in the area between neighboring holes and hence the gap between neighboring jetlets shear layers is smaller than a single holes annular shear layer diameter. As the shear layers spread, they interact with the neighboring shear layers before the closure of their own potential core. This causing increased turbulence (Figure 4e and f) and reduction in potential core length. Figure $4 \mathrm{a}$ and $\mathrm{b}$ illustrate the difference in the initial development of the fully merged jet plume. The increased mixing present on the outer most side of the outer jetlets, together with the partial holes (identified earlier) are seen to effect the jetlet trajectory and lead to an asymmetry in the fully merged jet plume. Figures 5a-f show how important the inclusion of the outer partial holes are in predicting the distortion they have on the overall mixed jet plume. Comparison between the distorted mixed jet plume to typically circular jet plumes must also play a part in the associated far-field noise. Given the number of permutations of outer circumferential hole geometries, this paper now focuses on the central hole, whose behavior is solely driven by the proximity of neighboring whole jetlets.

Comparison between the central jetlet of both the RANS and LES geometries for both porosities is shown in Figures 6 - 8. Figure 6 shows the increased potential core length commonly seen with RANS simulations. R40-2 can also be seen to posses an acceleration in centreline velocity at $x / D_{j}=20$. It is believed this increase in local velocity is due to higher energy fluid moving from the outlet region of the overall jet plume once the jetlet have become fully mixed. This is seen to its largest extent on the high porosity case due to the increased jetlet to jetlet interaction (illustrated in Figure 5e and f). The LES predictions are generally shorter than the RANS, however, in this case there is a distinct lack of potential core region in both LES simulations. The L40-2 jet axial velocity decays rapidly while the L23-2 decays at a slower rate to begin 


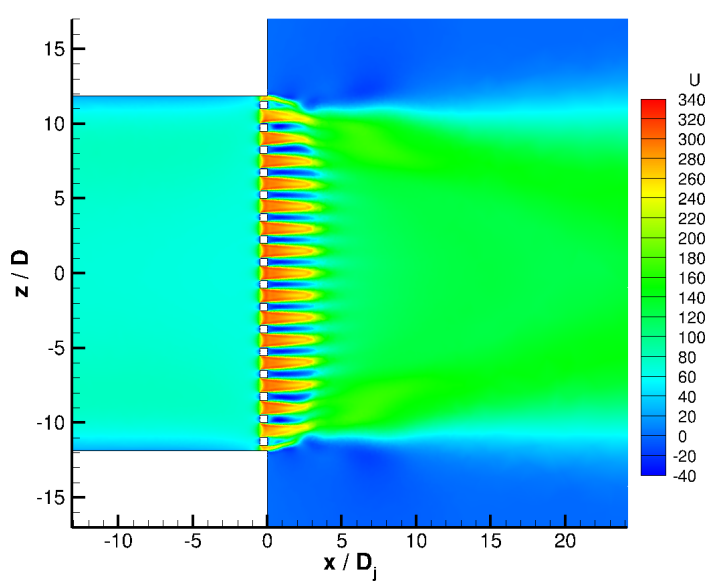

(a) R40-2 - Axial velocity for whole plate

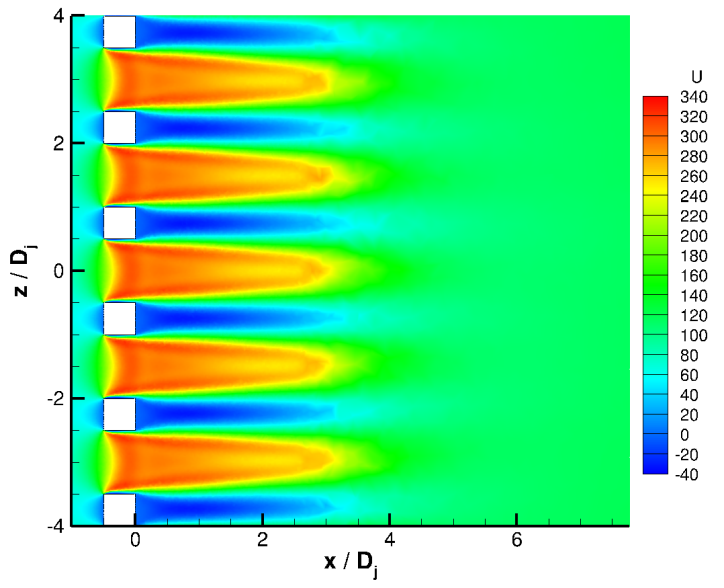

(c) R40-2 - Axial velocity for central holes

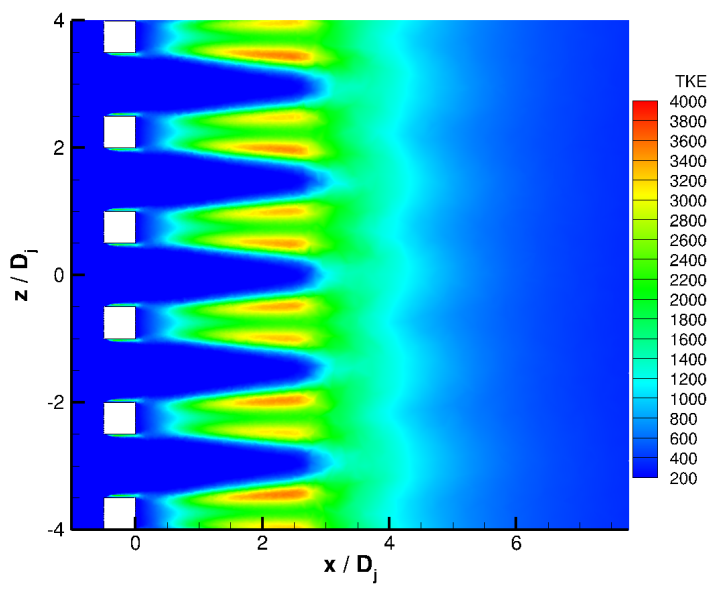

(e) R40-2 - tke for central holes

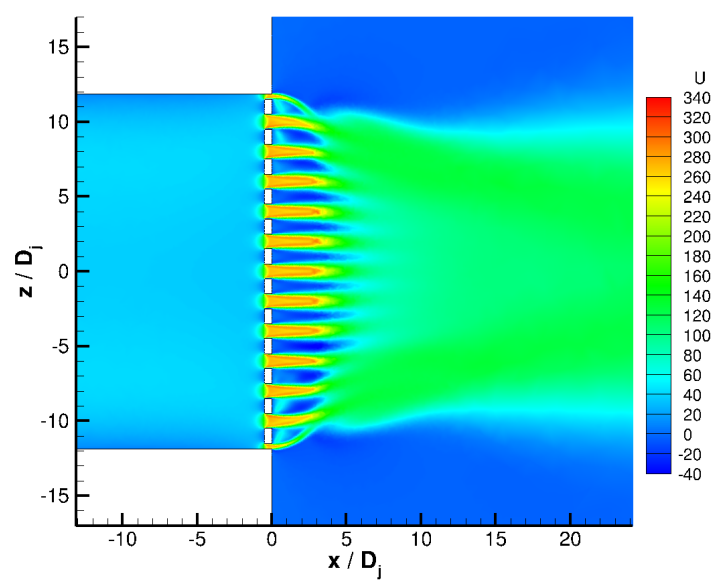

(b) R23-2 - Axial velocity for whole plate

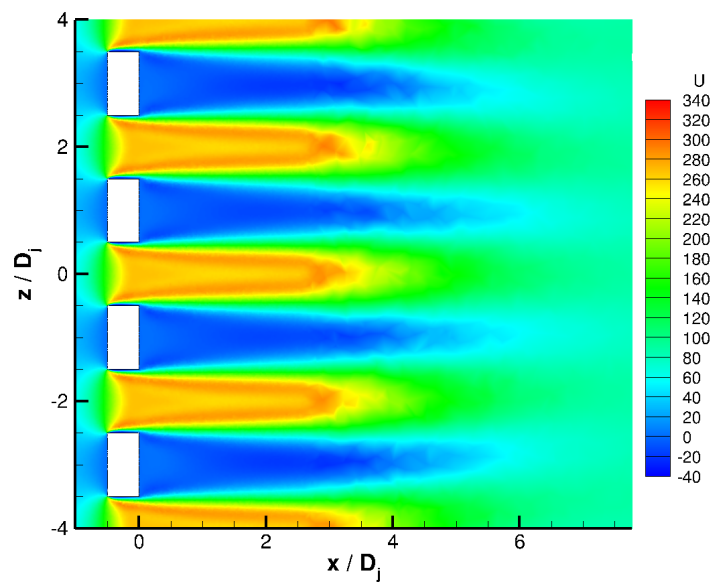

(d) R23-2 - Axial velocity for central holes

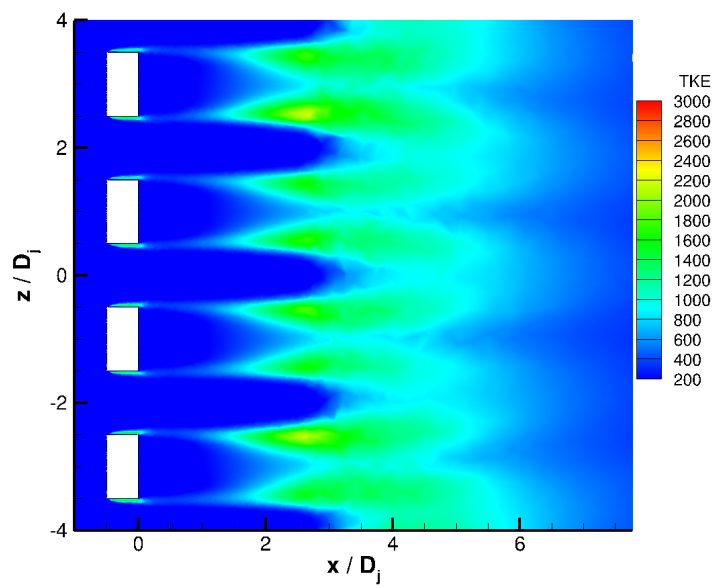

(f) R40-2 - tke for central holes

Figure 4: XY planes of axial velocity at various axial locations for $40 \%$ and $23 \%$ perforates 


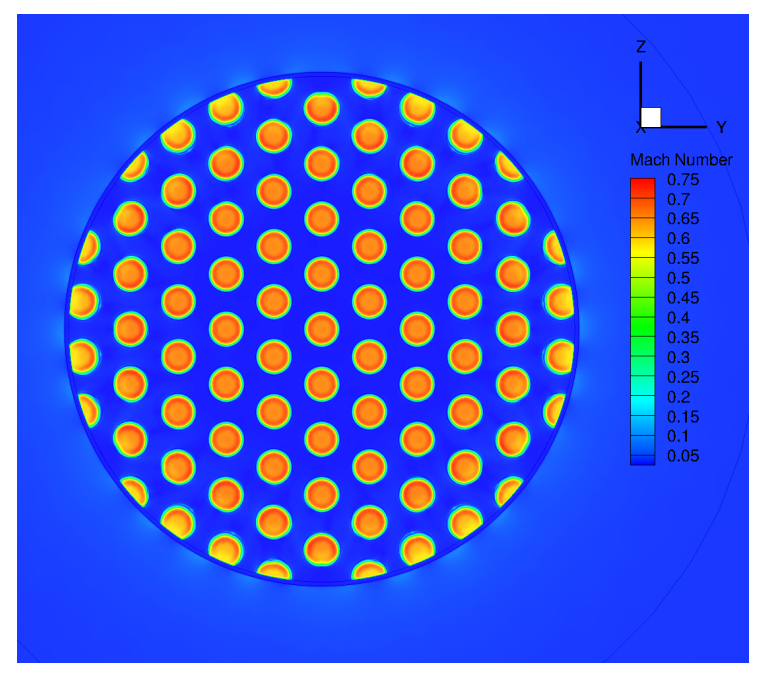

(a) $\mathrm{R} 40-2, \mathrm{x}=0.001 \mathrm{~mm}$

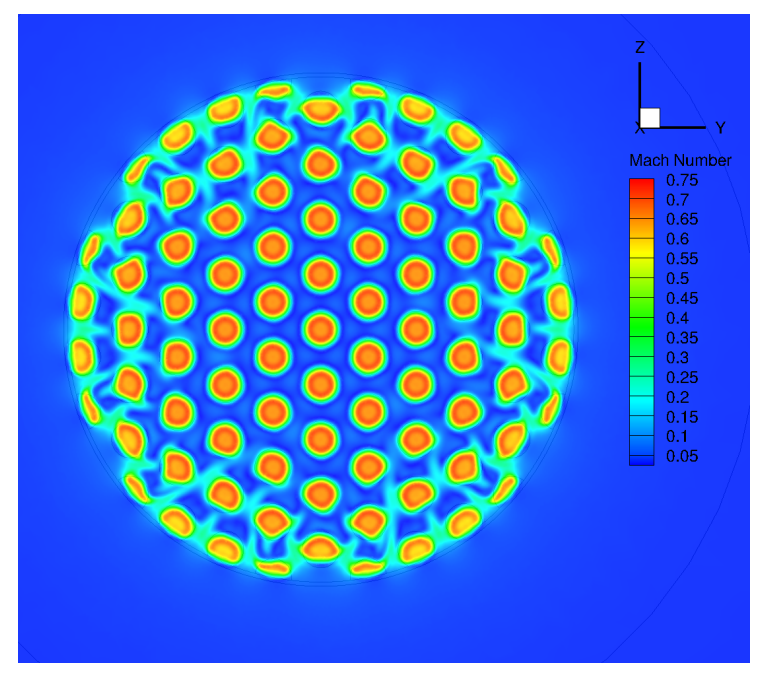

(c) $\mathrm{R} 40-2, \mathrm{x}=1.0 \mathrm{~mm}$

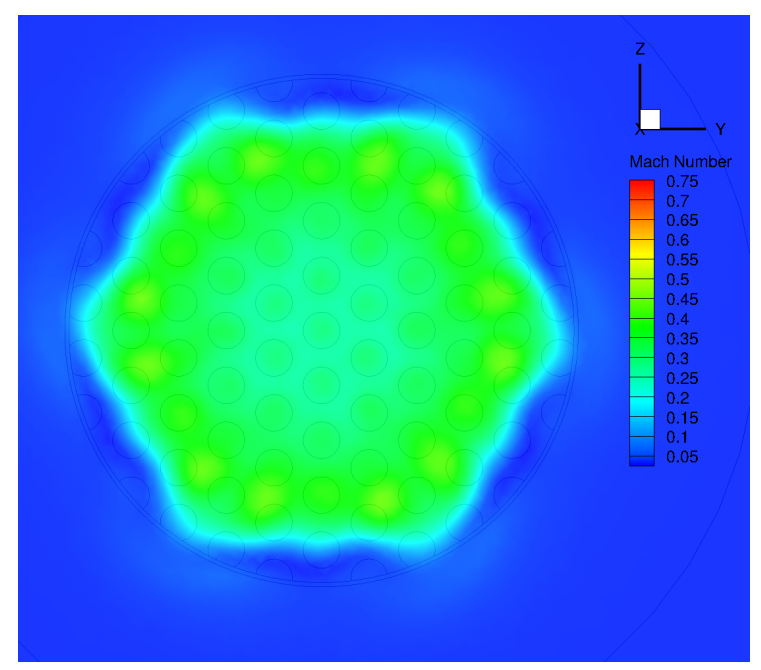

(e) $\mathrm{R} 40-2, \mathrm{x}=10.0 \mathrm{~mm}$

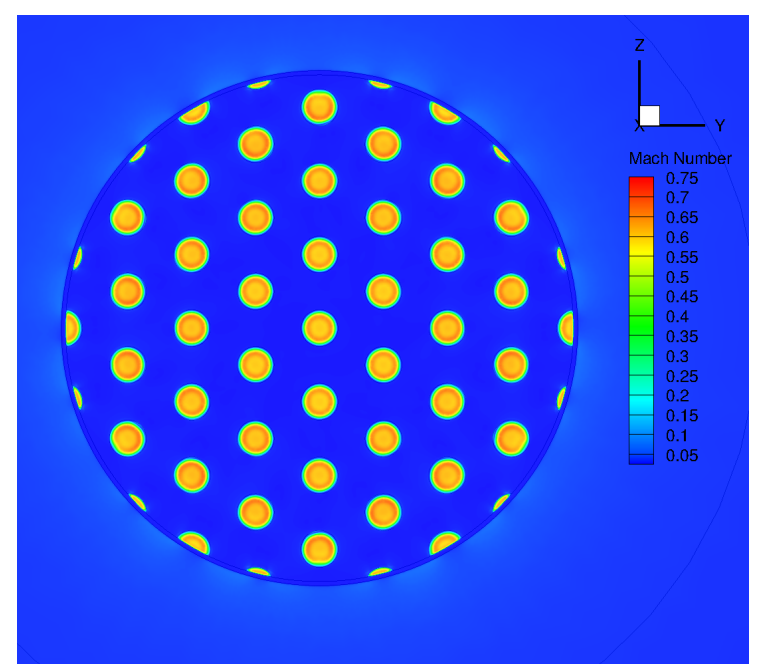

(b) $\mathrm{R} 23-2, \mathrm{x}=0.001 \mathrm{~mm}$

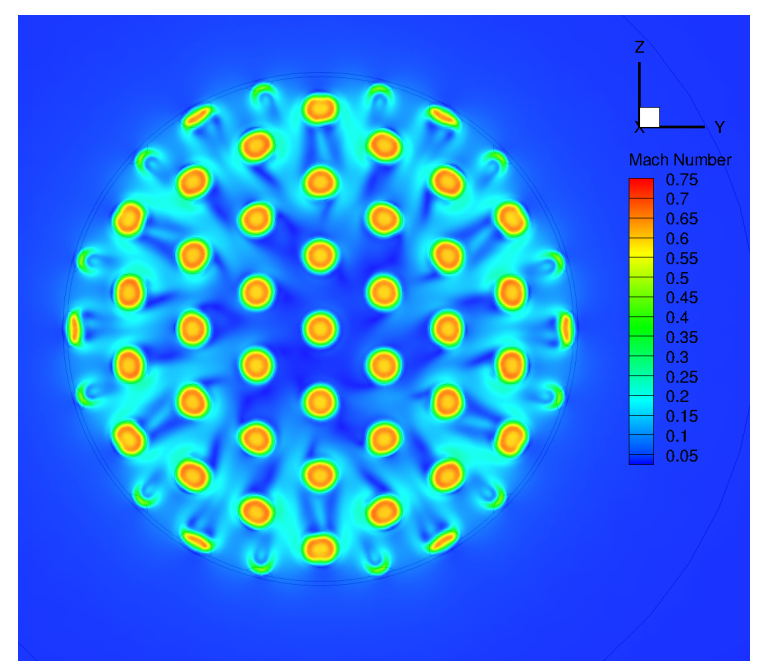

(d) R23-23, $x=1.0 \mathrm{~mm}$

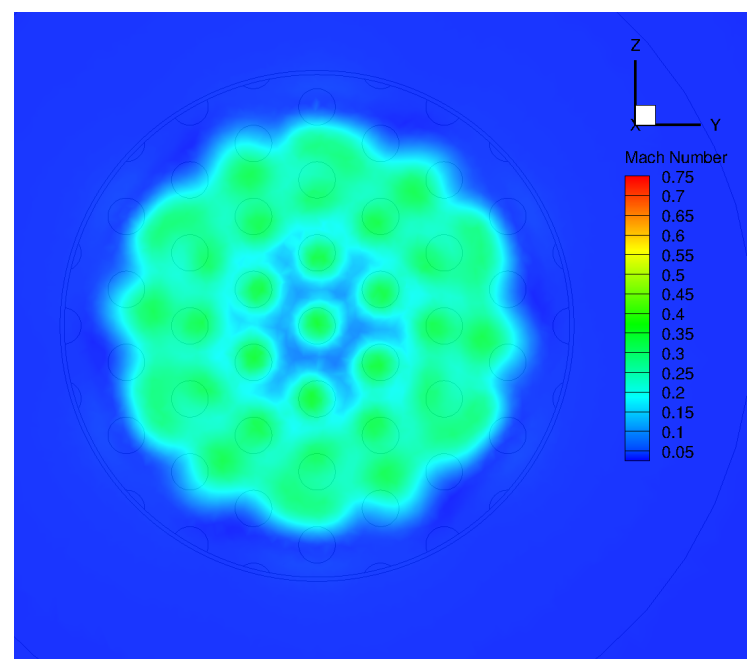

(f) $R 23-2, x=10.0 \mathrm{~mm}$

Figure 5: YZ planes of axial velocity at various axial locations for $40 \%$ and $23 \%$ perforates 
with, but by $x / D_{j}=3$ decays at the same rate as the L40-2 simulation. The L40-2 and L23-2 jetlet becomes fully mixed with its neighbouring jets at $x / D_{j}=4$ and $x / D_{j}=6$ respectively. The jetlet mixed region, produces an overall jet plume which posesses the typical plateau (steady velocity) region before the annular shear layer around the overall jet plume closes the potential core at approximately $x / D_{j}=55$ or $x / D p=4.8$ and $x / D_{j}=40$ or $x / D p=3.5$ for both L40-2 and L23-2 respectively. Note, the longer overall jet plateau for the $40 \%$ porosity plate is due to the larger effective overall jet diameter (based on open area diameter).

Figures 7 and 8 show profiles of the r.m.s of the velocity fluctuations. Downstream of $x / D_{j}=10$ the two simulations are comparable, the larger scale motions which have developed within the shear layer have overcome the earlier difference in plate porosity. When examining the r.m.s upstream of $x / D_{j}=10$ both the centreline and lipline profiles show a distinct two peak profile for the L23-2 simulations, whilst the L40-2 simulations shows a single peak. This single peak, although equal in magnitude to the twin peaks along the centreline, is higher along the lipline.

Figure 9a-b shows the radial profiles of axial mean and r.m.s velocity of the central jets. This shows the jetlet shear layers, due to the high porosity, interact with the neighbouring shear layers before the closure of its potential core. The L40-2 simulation has a negative velocity (recirculation zone) downstream of the plate. This results in higher r.m.s levels within the region of lee between the neighboring jetlet in comparison to the L23-2 simulation.

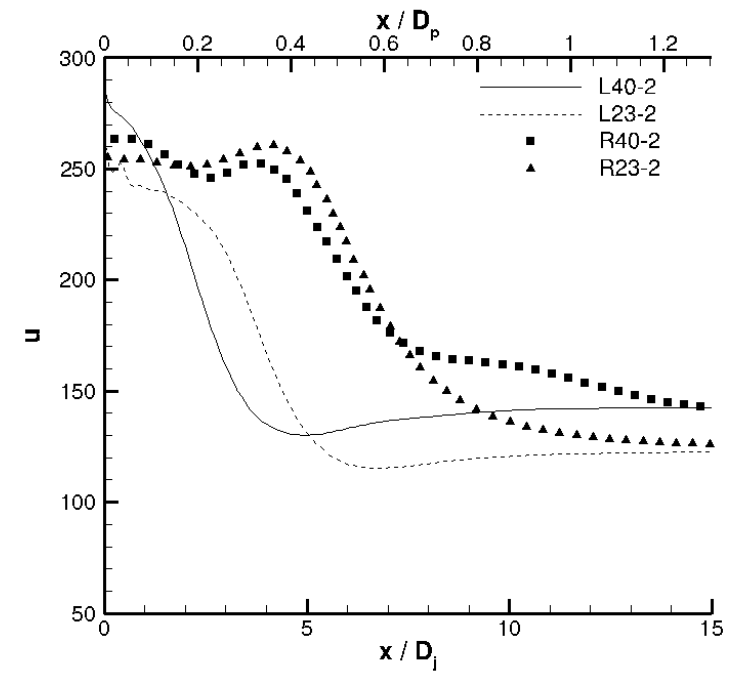

(a) Jetlet plume region

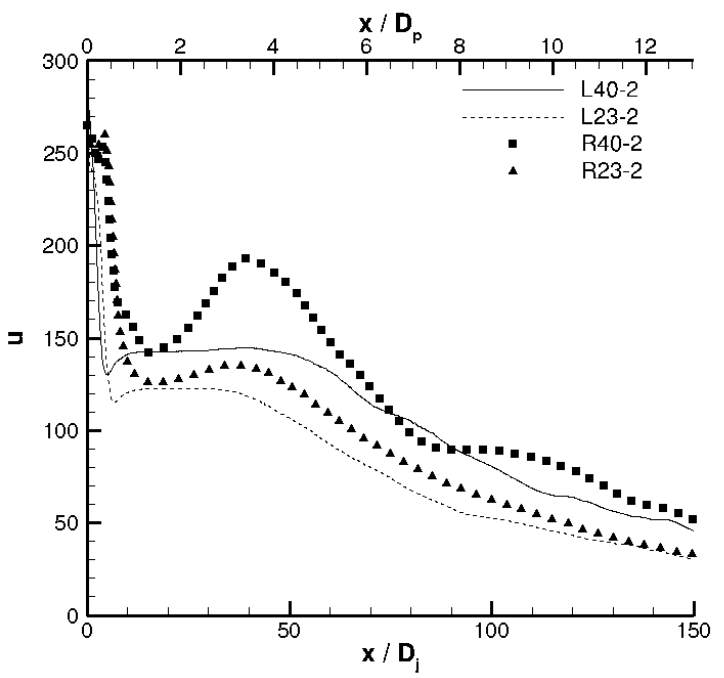

(b) Whole plume region

Figure 6: Comparison between RANS and LES axial profiles of axial mean velocity for both L40-2 and L23-2 predictions along the jet centreline

\section{B. Flow Structures}

A major advantage of LES is the ability to obtain fully time-dependent solutions allowing investigation into the fluid dynamics and presence of coherent structures in more detail. Instantaneous axial velocity and pressure contour plots for the whole jet plume and the central jetlet region are shown in Figures 10a-f for both L40-2 and L23-2 simulations. There is a noticeable difference for the two configurations in the shape and distribution of axial velocity through the hole and its dissipation into the overall jet plume. The regions of low pressure in Figure 10e and f signify the presence of shear layer vortical structures. As the jetlet emerges from the plate in the $23 \%$ case, the vortical structures are aligned across the shear layer and aligned with the adjacent jetlets (as will be seen later, there are toroidal (ring) structures). For the $40 \%$ case the low pressure indicates the vortical structures are staggered across the jetlet and staggered in respect to the adjacent jetlets. (Representative visualisations of the vortical structures in the flow are shown in Figures 11 and 12 for both L23-2 and L40-2 simulations). Visualisation using an isosurface of vorticity magnitude 


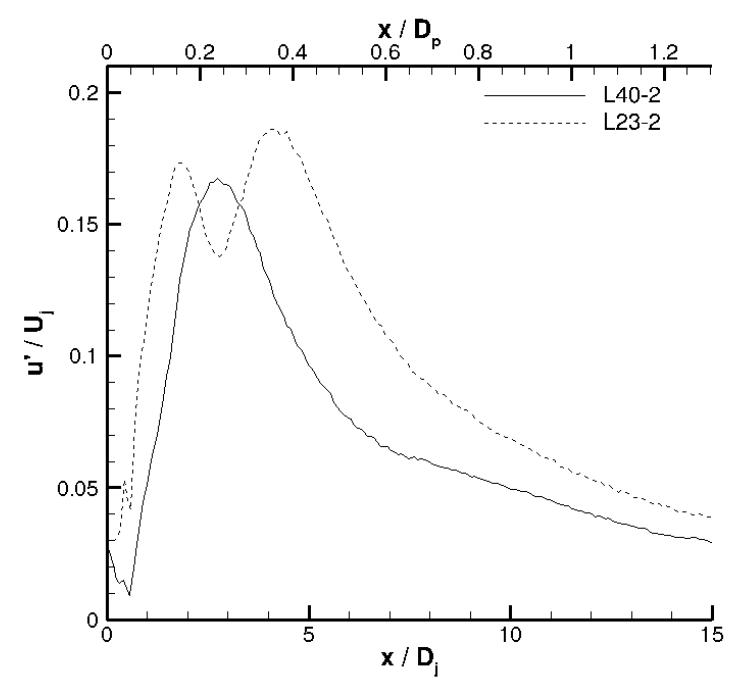

(a) Jetlet plume region

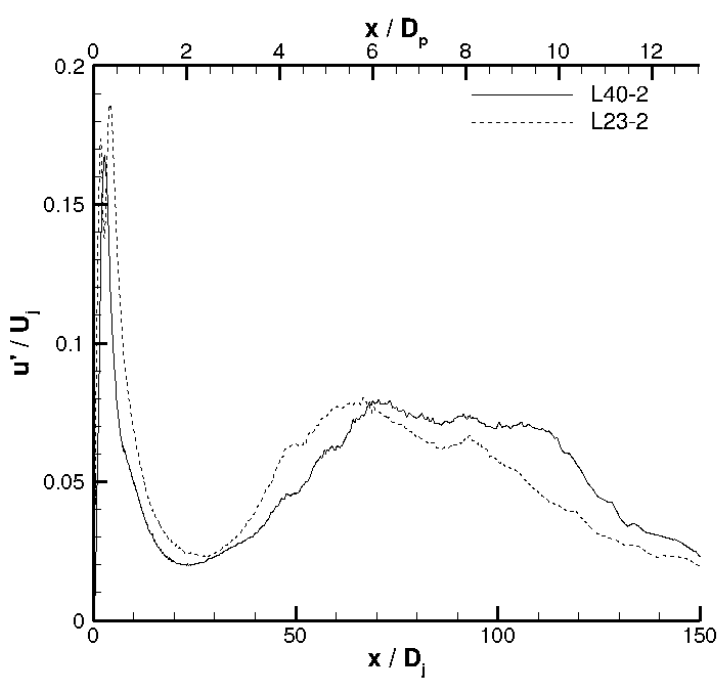

(b) Whole plume region

Figure 7: Axial profiles of axial r.m.s velocity for both L40-2 and L23-2 predictions along the jet centreline

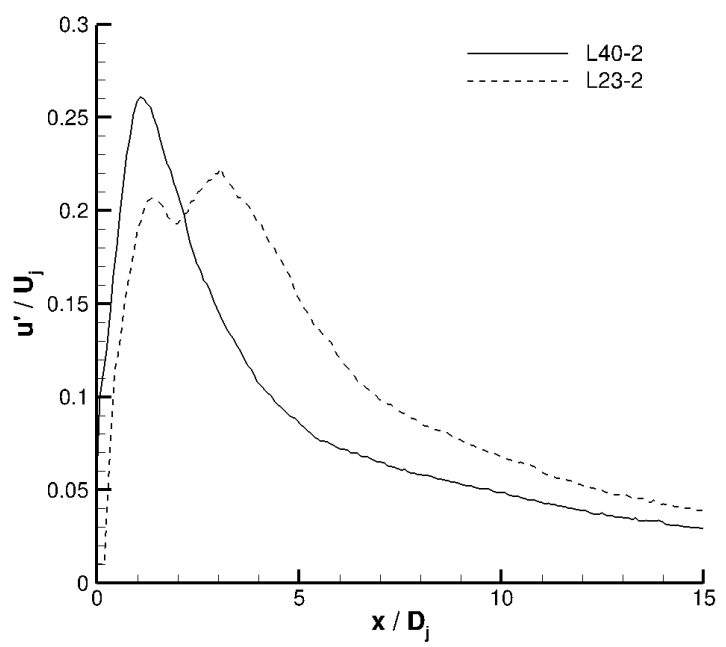

(a) Jetlet plume region

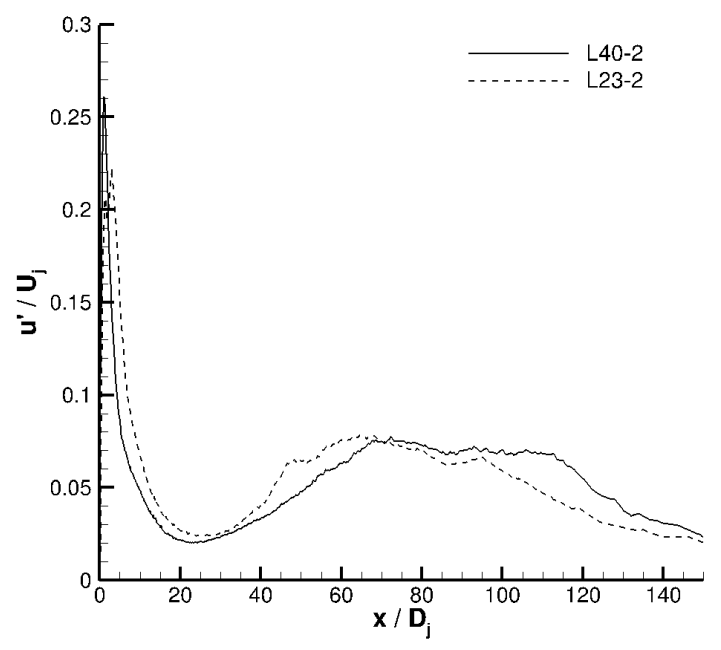

(b) Whole plume region

Figure 8: Axial profiles of axial r.m.s velocity for both L40-2 and L23-2 predictions along the central hole lipline 


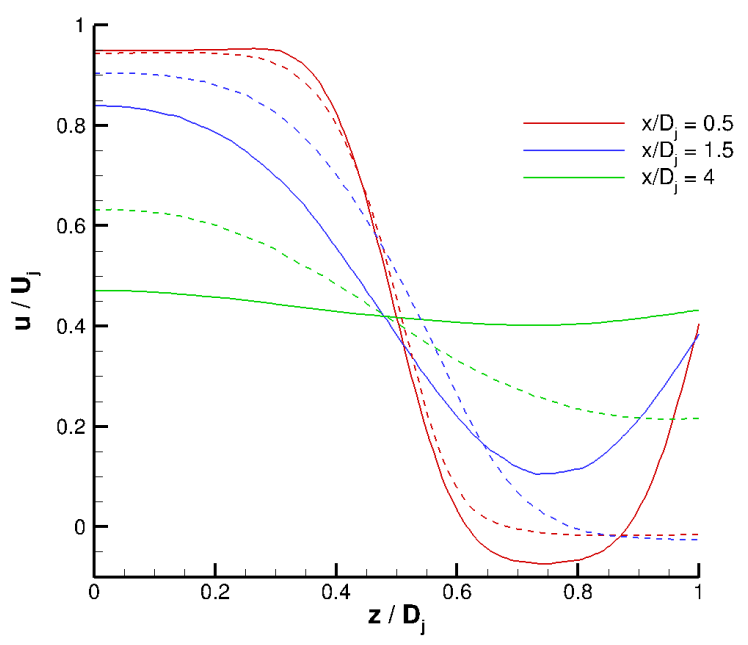

(a) Jetlet plume region

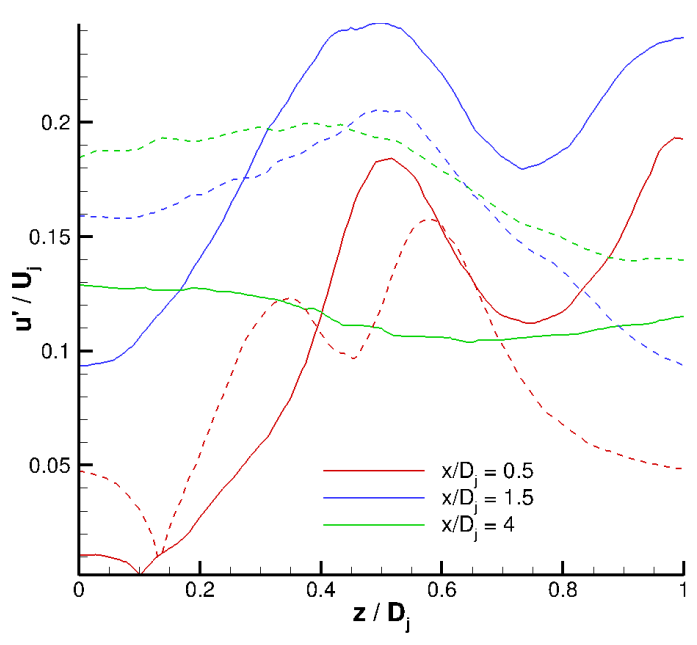

(b) Whole plume region

Figure 9: Radial profiles of axial mean and r.m.s velocity for both L40-2 and L23-2 predictions (solid lines $=\mathrm{L} 40-2$, dashed lines $=\mathrm{L} 23-2$ )

(Figure $11 \mathrm{~b}$ and $12 \mathrm{~b}$ ) show vortex ring structures typical of a jet with laminar initial condition ${ }^{16}$ and with some similarity to the vortex train produced by a synthetic jet. In the $40 \%$ porosity perforate case, the reduced distance between the holes leads to increased interaction between the jetlets resulting in a dominant helical vortex structure and additional small scale turbulence. These structures also increase the turbulence levels and reduces the potential core length of the jetlets.

The different vortex structures is likely to be due to the ambient air entrainment process of the jetlets. Each jetlet requires a certain amount of mass to be entrained to be able to expand like an individual, undisturbed one. In this case, the air would be delivered from the ambient environment but due to the small spacing between two adjacent holes, insufficient fluid is available to feed the jetlets. This results in a low pressure region - although not constant in time. Regarding the temporal development of the inner hole region, it can be seen that a separation bubble emerges due to the sharp inlet edge causing a reduction in the effective flow area. Due to the lack of entrainment air in the region of lee downstream of the plate, the separation bubble is sucked out of the hole and into this low-speed lee region to provide the required mass flow. The instantaneous mass compensation leads to a breakdown of the driving force for the suction of the separation bubble. Hence a new one is formed and the mechanism starts again. This process happens for all holes at the same time. Therefore, an equilibrium is achieved between suction of the separation bubble to provide enough fluid in the lee regions and the instantaneous entrainment of this air. For the L40-2 case, the close proximately of neighboring jetlets results in the formation of an entrainment region roughly opposite of it located suction region which circulates around the centerline of each jet. Superimposed with the axial velocity of the jetlets, this motion leads to the occurrence of the helical vortex structures. 


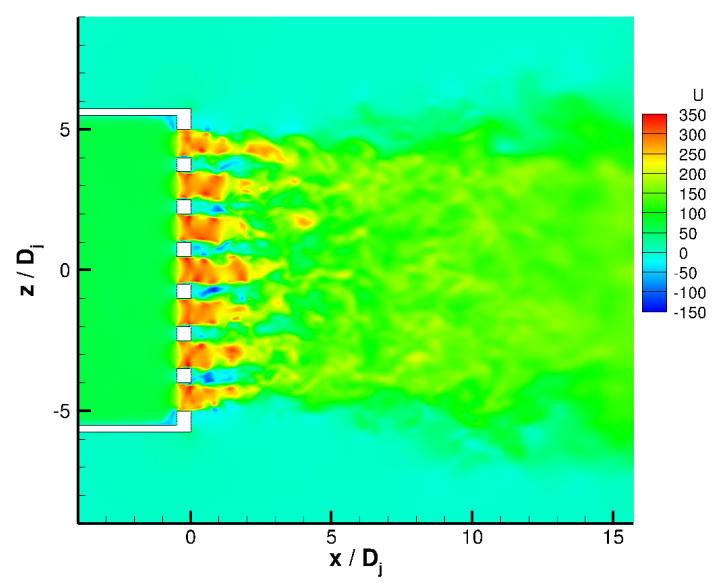

(a) L40 - Axial Velocity Contours

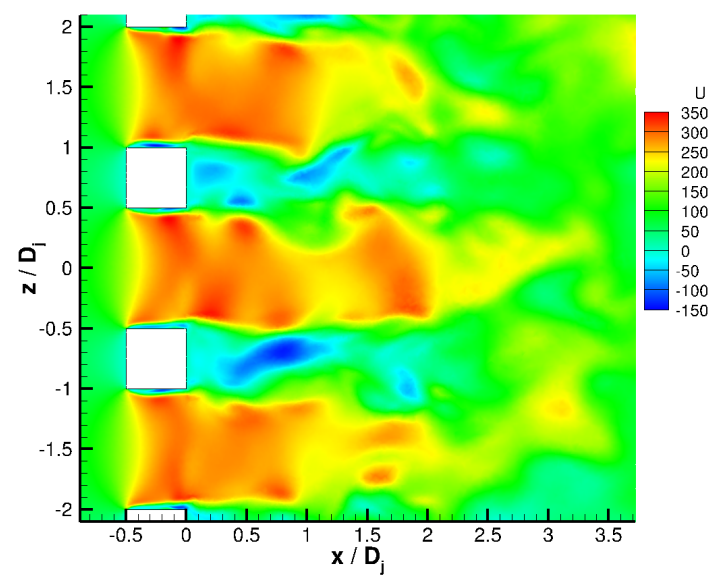

(c) L40 - Axial Velocity Contours

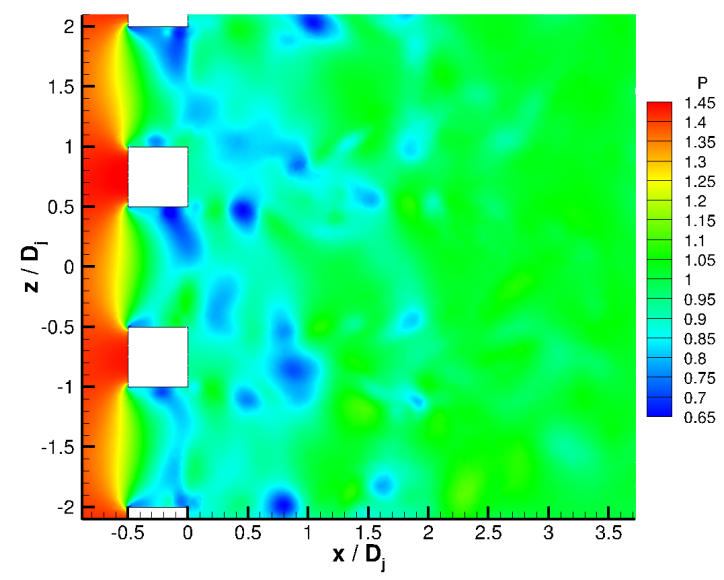

(e) L40 - Pressure Contours

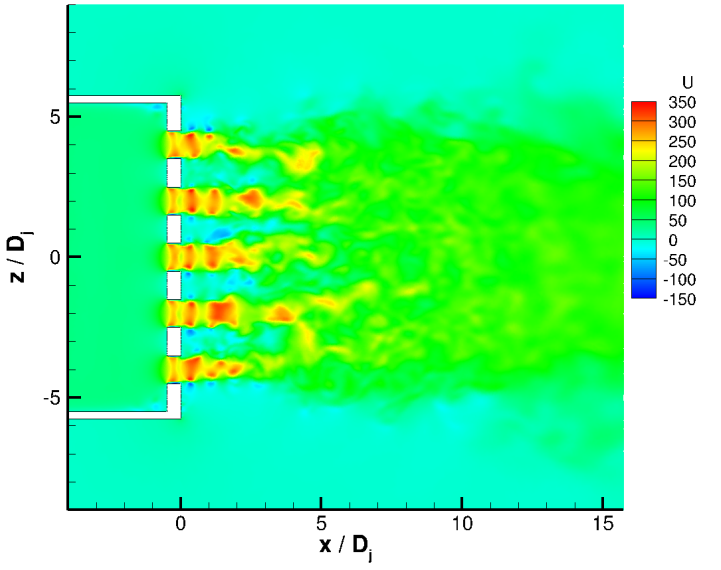

(b) L23 - Axial Velocity Contours

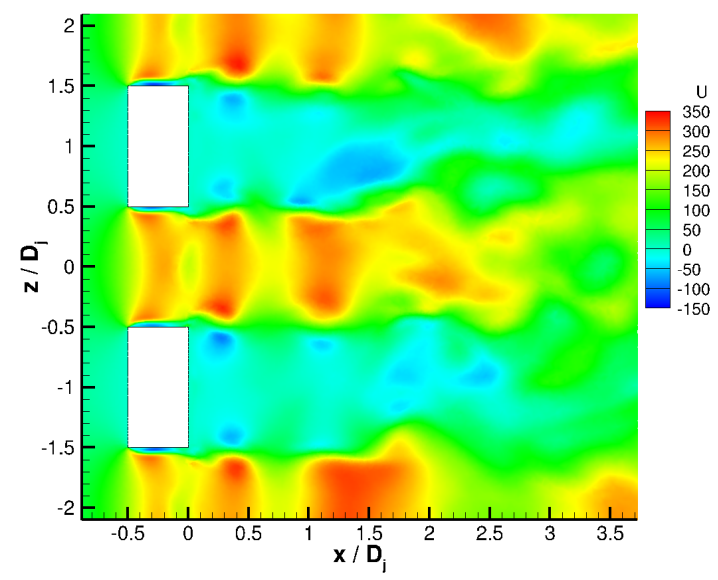

(d) L23 - Axial Velocity Contours

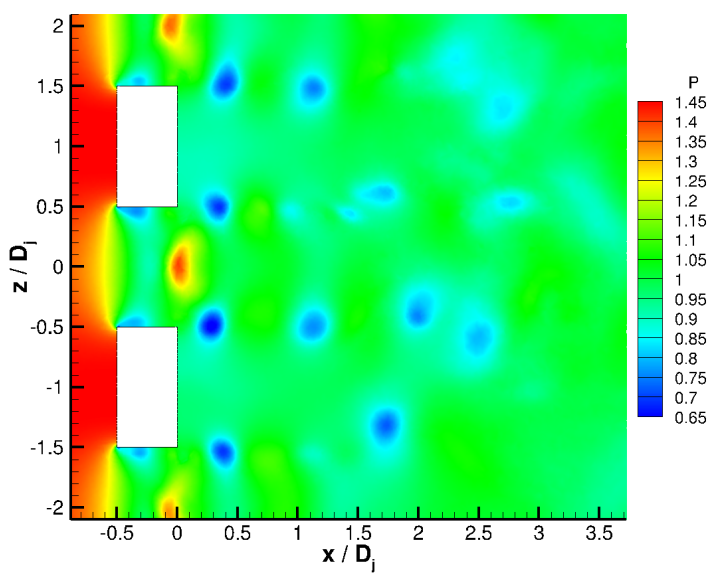

(f) L23 - Pressure Contours

Figure 10: XZ planes of Mach number at $\mathrm{Y}=0$ for $\mathrm{T} 40$ and $\mathrm{T} 23$ perforates 


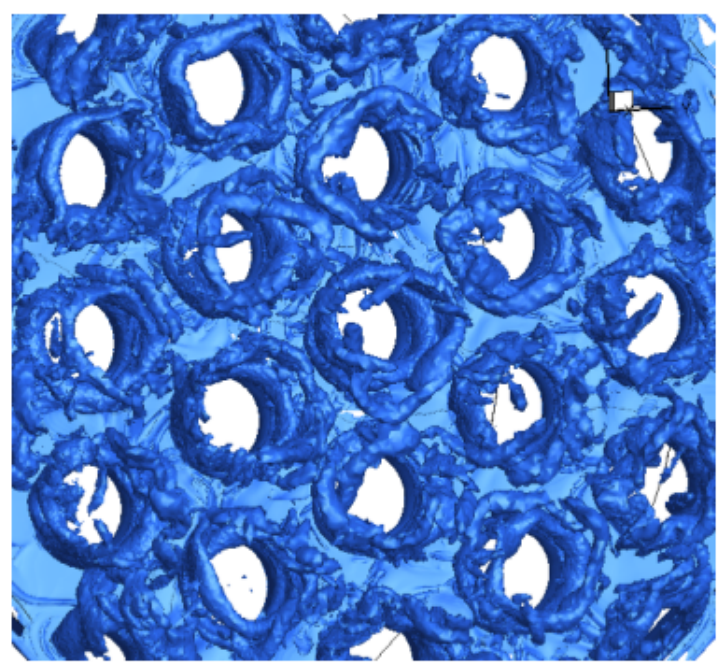

(a) $40 \%$ porosity

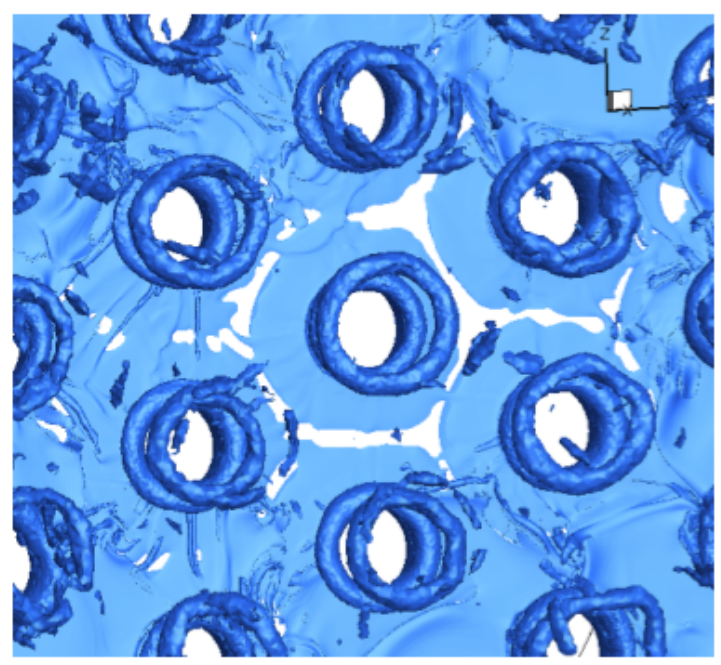

(b) $23 \%$ porosity

Figure 11: Iso-surfaces of $\Omega_{r e l}=2.7 \%$ at the central plate area for both geometries
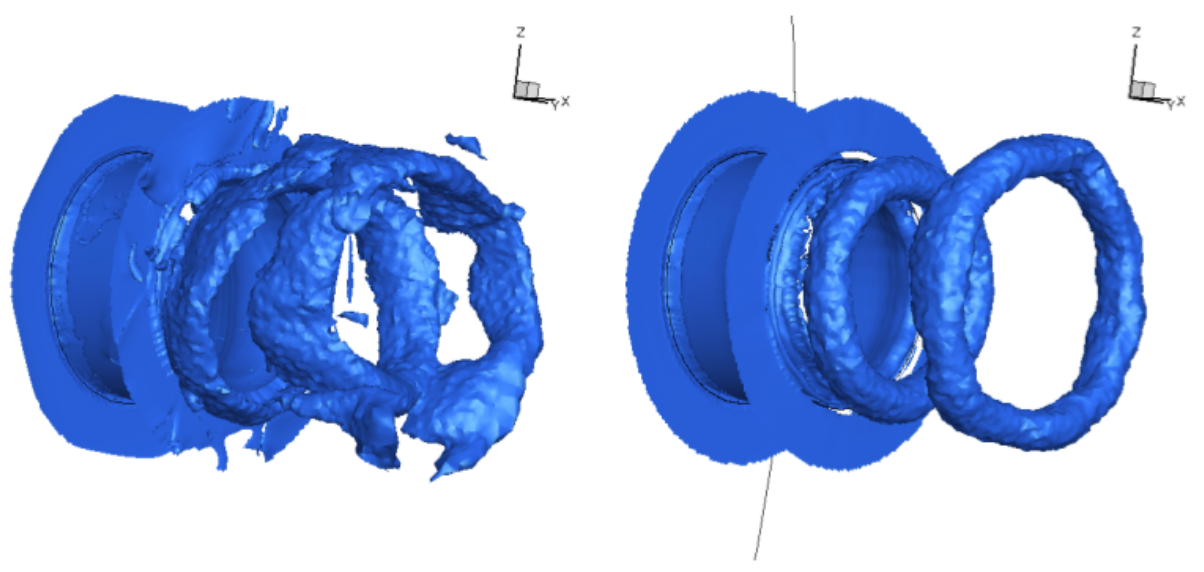

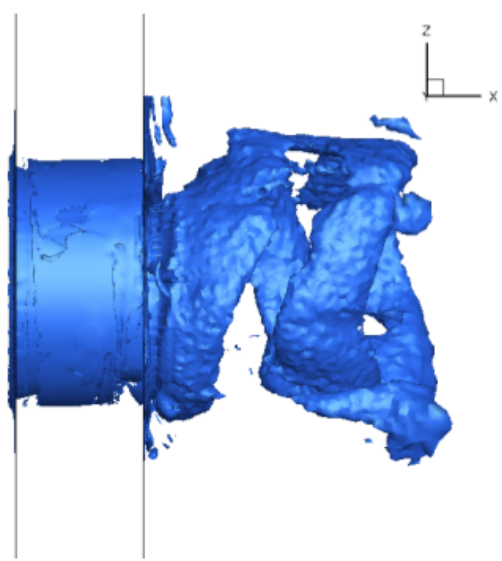

$40 \%$ porosity

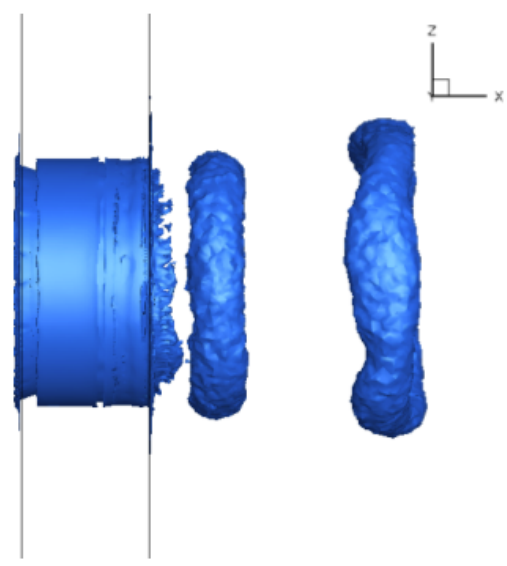

$23 \%$ porosity

Figure 12: Iso-surfaces of a relative vorticity magnitude of $\Omega_{r e l}=3.6 \%$ at the most central hole for both geometries seen from two different perspectives 


\section{Correlations}

In order to understand the acoustic sources the two LES solutions have been post-processed to extract the 2-point 2-time correlations for both $2^{\text {nd }}$ and $4^{\text {th }}$ order statistics, as defined in equations 17 and 18 . The correlations are evaluated at a point in the middle of the shear layer $\left(r / D_{j}=0.5\right)$. The auto-correlation function is obtained by setting $\vec{\eta}=0$ and gives rise to the local integral timescale. Figures 13 illustrates the auto-correlation at $x / D_{j}=1.5$ and $x / D_{j}=4$. The second peak value in both $2^{\text {nd }}$ and $4^{\text {th }}$ correlations for both L40-2 and L23-2 represents the passing of a correlated structure (i.e the next vortex ring). The peaks occur at approximately the same temporal decay indicating that the frequency is similar in both cases, as would be expected as this is generally a driven by a geometric length and characteristic velocity.

As expected the secondary peak magnitude is larger for the L23-2 case due to the highly coherent nature of multiple vortex rings, in comparison to a helical structure interlaced with high frequency structures. Figure $13 \mathrm{c}$ illustrates the same secondary peak correlations at $\tau 0.6 U_{j} / D_{j}=2$ whilst also bringing to attention an earlier smaller peak only present in the L23-2 prediction. This tertiary peak is associated with the secondary streamwise vortices which straddle the two rolling vortex rings and produce a region of mildly correlated axial velocity fluctuations. The lack of secondary peaks for both simulations at $x / D_{j}=4$ illustrates that by this axial location both structures have undergone transition into a fully turbulent flow.

Figure 14 and 15 shows the $2^{\text {nd }}$ and $4^{\text {th }}$ order correlations for the axial velocity fluctuation $\left(R_{11}\right)$ and for varying axial separation and delay time. For all separation distances $(\eta)$ a noticeable secondary peak exists for all velocity components (Axial - R11, Radial - R22, and Circumferential - R33). The decay can be seen to be slower for the L23-2 simulations associated with the low level of diffusion in vortex rings present at $x / D_{j}=1.5$. The evolution of the shear layer development with axial distance results in an increase in decay rate for L23-2 in comparison to L40-2 by $x / D_{j}=4$. This is associated by the rapid transition to fully-developed turbulence downstream of vortex ring breakdown. ${ }^{16}$

To address the question of relative amplitudes of correlation components, the peak magnitude (numerator) of all $62^{\text {nd }}$ order and $214^{\text {th }}$ order independent correlation components for zero time and space separations normalised by the axial component peak magnitude at $x / D_{j}=1.5, z / D_{j}=0.5$ are examined. Table 2 shows a comparison of $2^{\text {nd }}$ order relative correlation amplitudes between the PIV results from Pokora et al, ${ }^{17} \mathrm{CTA}$ results from Harper-Bourne ${ }^{18}$ and the two LES simulations in this study. The agreement between the LES simulations is good for all terms except $R_{33}$ where the helical nature of the flow structure produced by the L40-2 geometry increase the circumferential term. The non-isotropic 2D coherent structures can be clearly seen in both results in the difference between $R_{22}$ and $R_{33}$.

\begin{tabular}{c|cccccc} 
& $\mathbf{R}_{\mathbf{1 1}}$ & $\mathbf{R}_{\mathbf{1 2}}$ & $\mathbf{R}_{\mathbf{1 3}}$ & $\mathbf{R}_{\mathbf{2 2}}$ & $\mathbf{R}_{\mathbf{2 3}}$ & $\mathbf{R}_{\mathbf{3 3}}$ \\
\hline Pokora $^{17}$ PIV data & 1 & 0.30 & -0.04 & 0.42 & 0.03 & 0.67 \\
Harper-Bourne $^{18}$ CTA data & 1 & - & - & 0.61 & - & 0.80 \\
L23-2 LES data & 1 & 0.47 & -0.03 & 1.01 & 0.04 & 0.34 \\
L40-2 LES data & 1 & 0.48 & -0.04 & 0.92 & -0.02 & 0.83 \\
\hline
\end{tabular}

Table 2: Comparison of $2^{\text {nd }}$ order correlation component amplitudes as a ratio of $R_{11}$ between average PIV results and CTA results at $x / D_{j}=1.5, z / D_{j}=0.5$ ) 


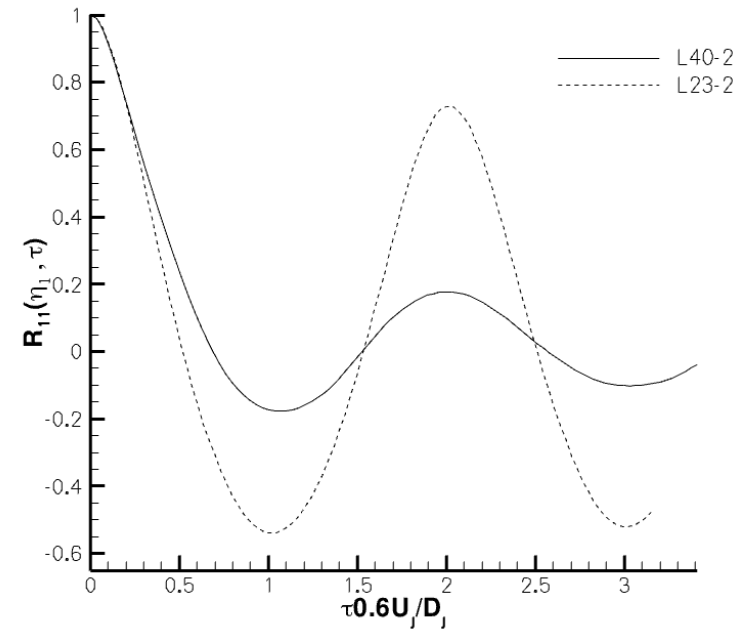

(a) $x / D_{j}=1.5$

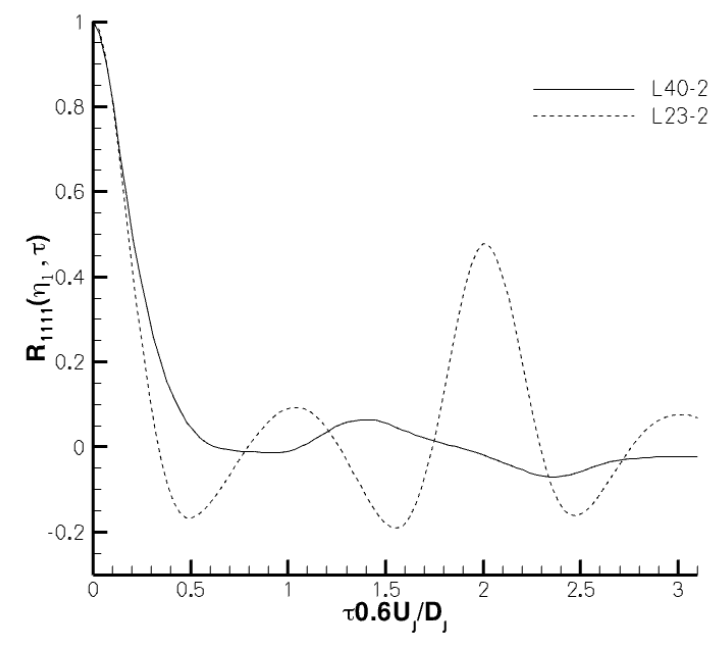

(c) $x / D_{j}=1.5$

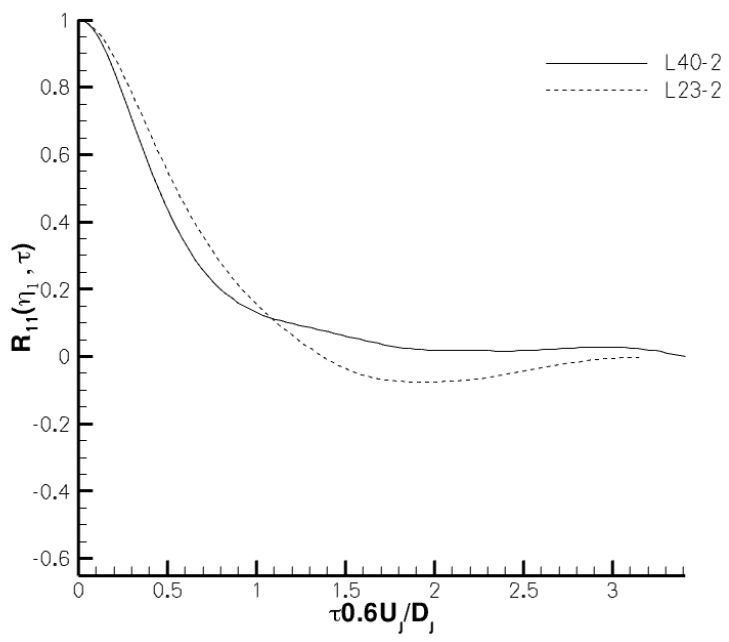

(b) $x / D_{j}=4$

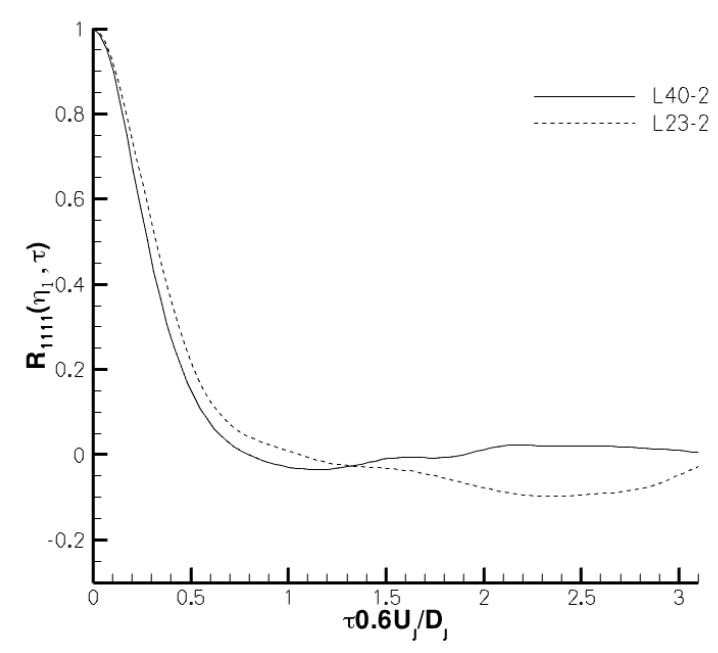

(d) $x / D_{j}=4$

Figure 13: Comparison between L40-2 and L23-2 for $2^{\text {nd }}$ and $4^{\text {th }}$ order auto-correlations of axial velocity fluctuations $(\eta=0)$ 


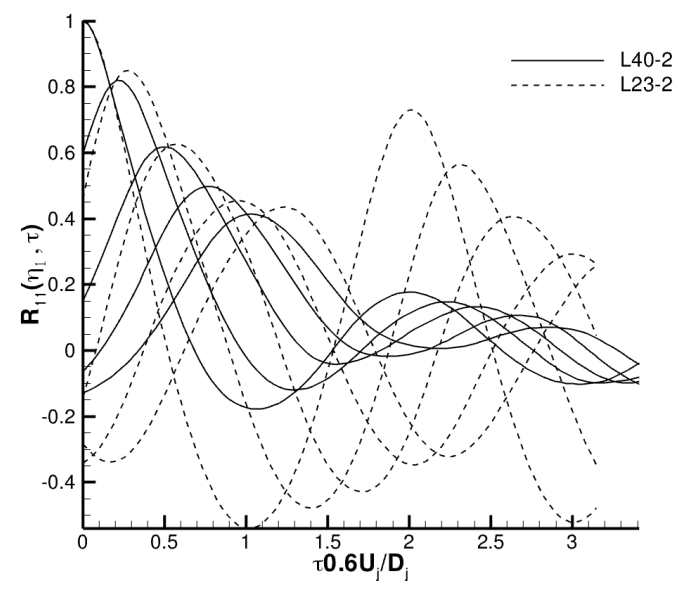

(a) $x / D_{j}=1.5$

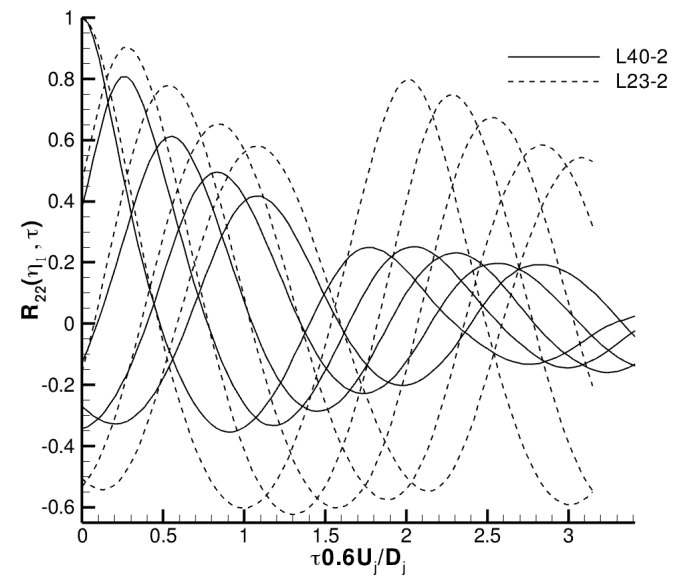

(c) $x / D_{j}=1.5$

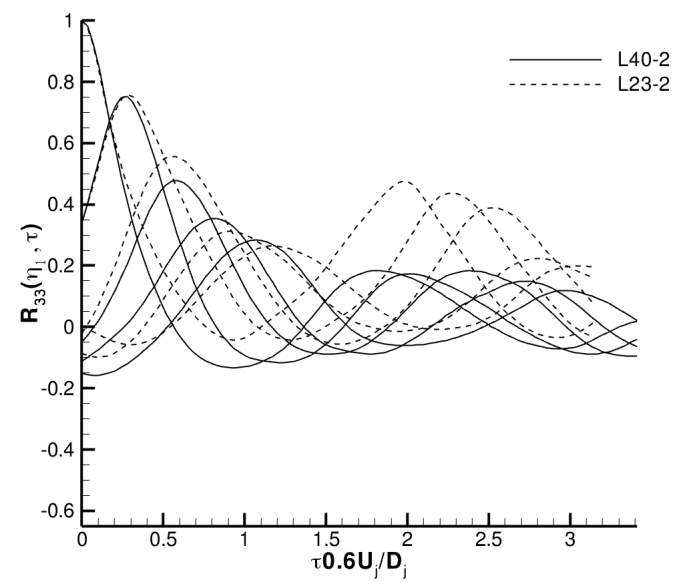

(e) $x / D_{j}=1.5$

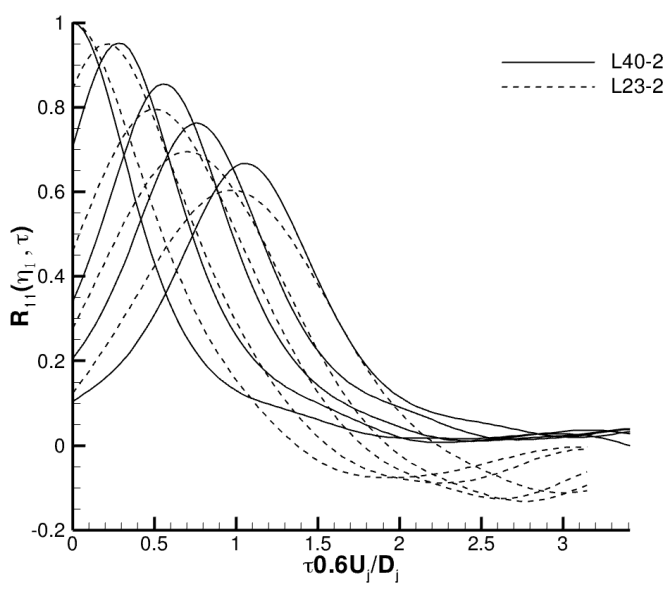

(b) $x / D_{j}=4$

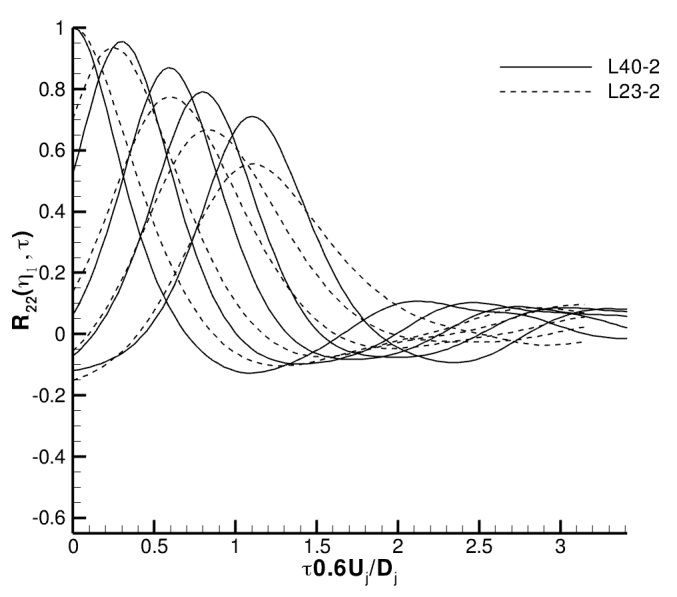

(d) $x / D_{j}=4$

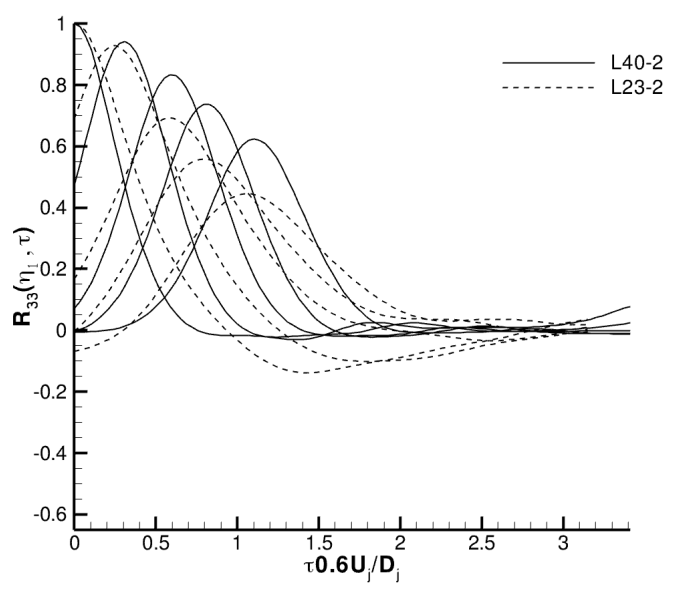

(f) $x / D_{j}=4$

Figure 14: Comparison between L40-2 and L23-2 for $2^{\text {nd }}$ order 2-point 2-time correlations of axial velocity fluctuations with axial separations (each line represents $\eta_{1} / D_{j}=0.2$ ) 


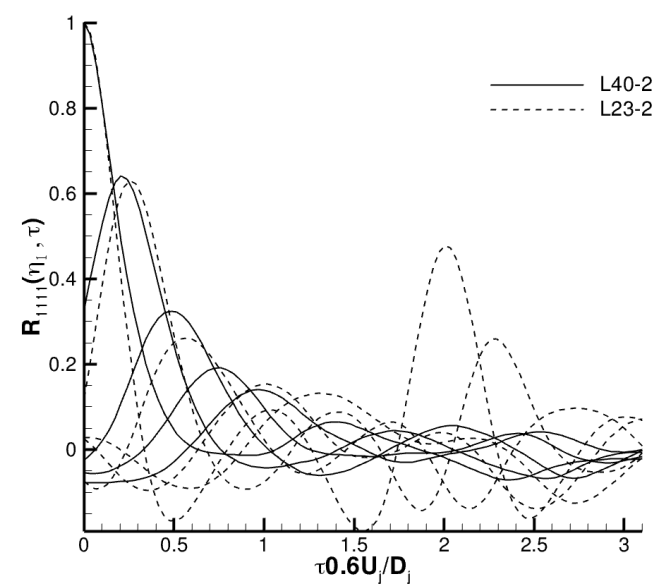

(a) $x / D_{j}=1.5$

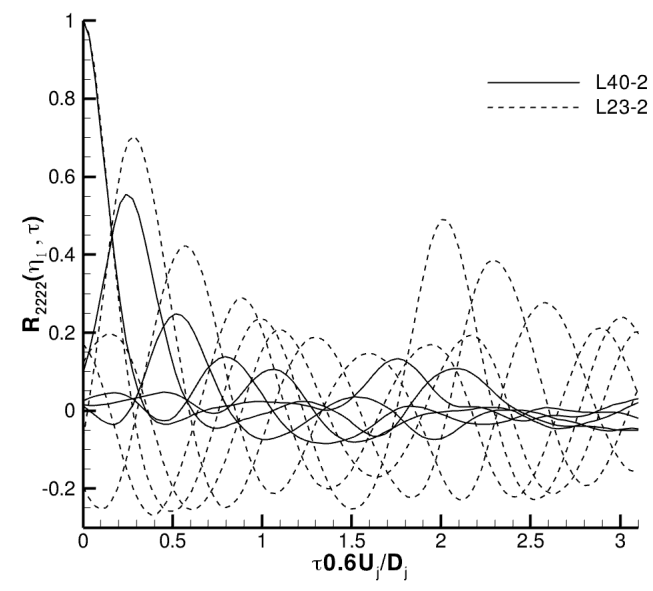

(c) $x / D_{j}=1.5$

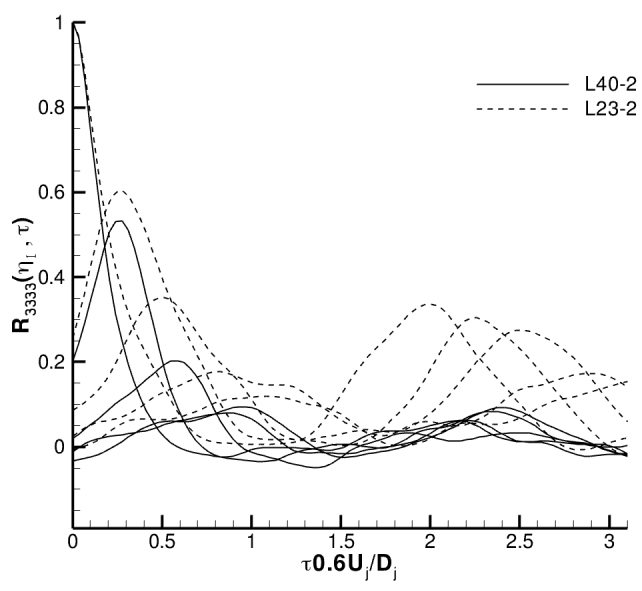

(e) $x / D_{j}=1.5$

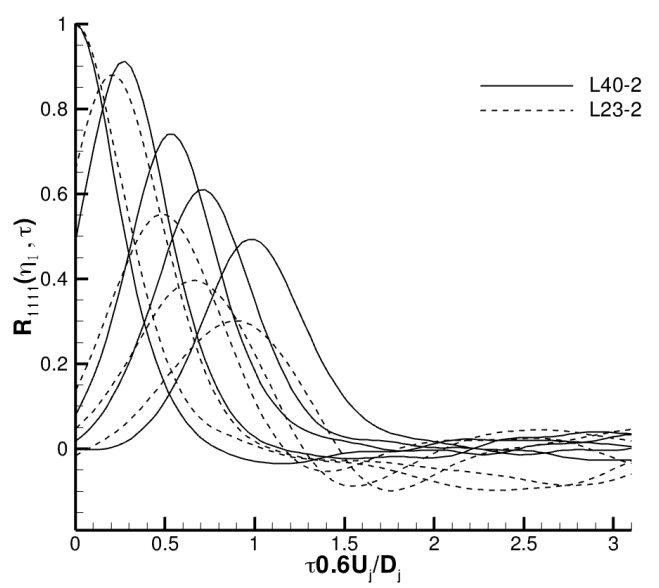

(b) $x / D_{j}=4$

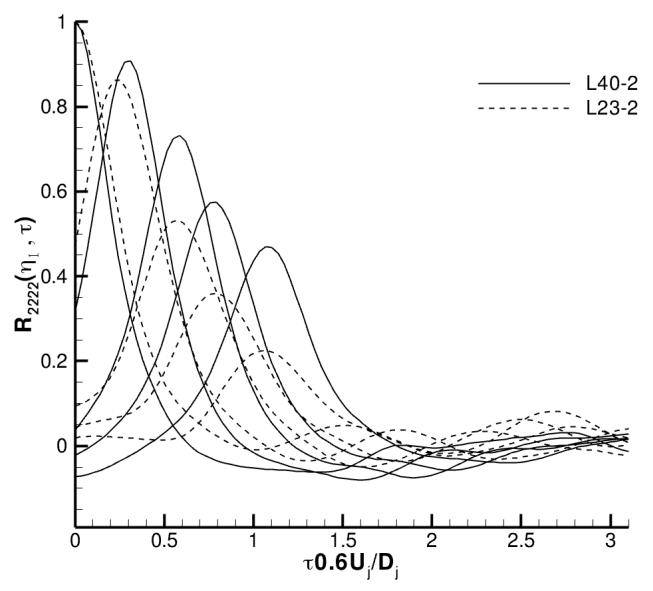

(d) $x / D_{j}=4$

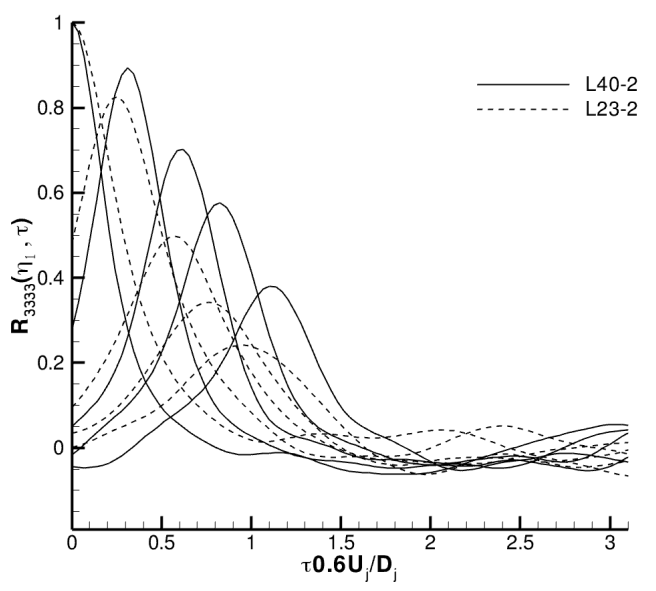

(f) $x / D_{j}=4$

Figure 15: Comparison between L40-2 and L23-2 for $4^{\text {th }}$ order 2-point 2-time correlations of axial velocity fluctuations with axial separations (each line represents $\eta_{1} / D_{j}=0.2$ ) 


\section{Far-field Noise Results}

To address the issue of acoustic source modeling together with noise levels in the far-field a Ffowcs-Williams \& Hawkings surface integral method was used. This enables a level of understand to be acquired about how the relative differences in the near field structures affect the far-field. It should be noted that the aim is not to evaluate the ability of the Ffowcs-Williams \& Hawkings surface integral method to predict accurately the far-field sound pressure levels (limited sample sizes make this impossible), but to use it as a tool to look at changes in the acoustic signal.

Figure 16 presents the power spectral density at observer angles $\theta=0^{\circ}, 45^{\circ}, 90^{\circ}$ and $120^{\circ}$ relative to the jet axis for both porosity perforates. Signal noise can be seen across the whole spectra and the limited sample size has resulted in poor low frequency results. However, there is a noticeable difference in the two signals in the high frequency range (the frequency expected to be affected by the small high frequency structures in the jetlet shear layer).

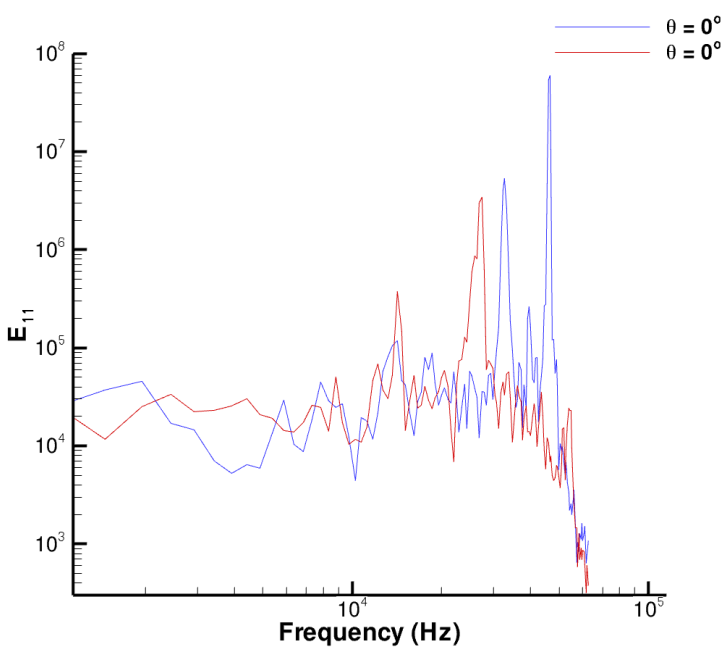

(a) $\theta=0$

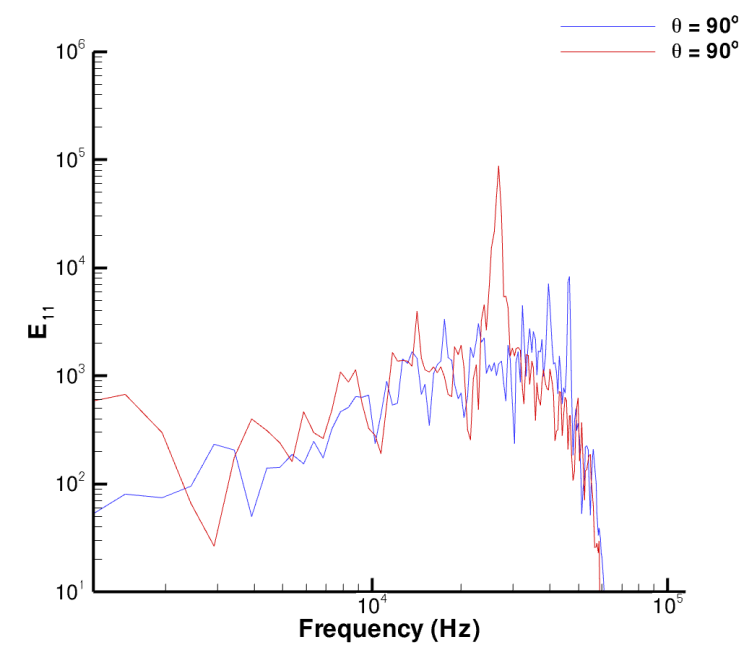

(c) $\theta=90$

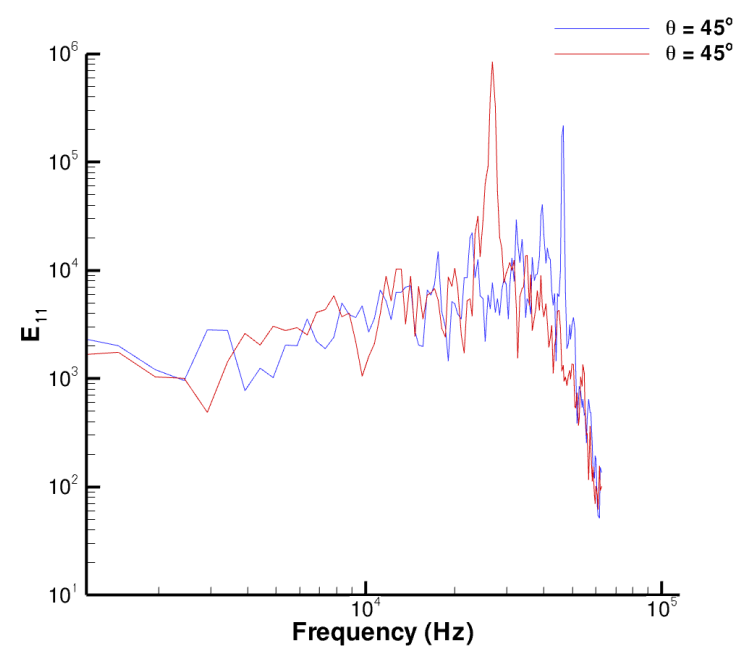

(b) $\theta=45$

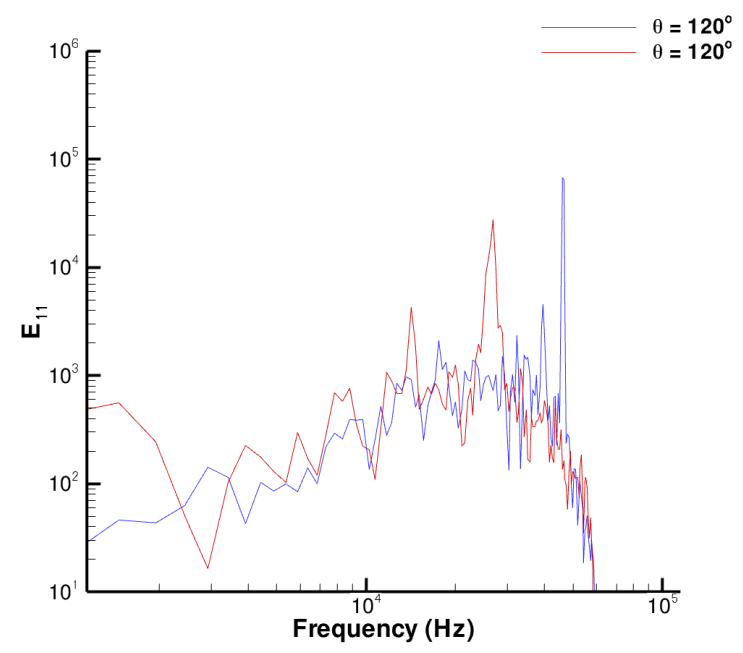

(d) $\theta=120$

Figure 16: Comparison of PSD between L40-2 (red) and L23-2 (blue) prediction from FWH surface integral at various observer angles 
The $23 \%$ and $40 \%$ porosity perforate shows the presence of a single dominant high energy high frequency peak at all observer angles at $\approx 46 \mathrm{kHz}$ and $\approx 27 \mathrm{kHz}$ respectively. The $23 \%$ porosity perforate peak frequency is consistent with a Strouhal number of $\approx 0.3$, based upon the jetlet diameter and velocity, where as for the $40 \%$ perforate produces a Strouhal number of $\approx 0.6$. The difference in the far field pressure signal illustrates that the presence of these fundamentally different near field flow structures and associated acoustic source is important for noise modeling as their influence can be seen in the far-field pressure signal.

\section{Conclusion}

The study has shown that the RANS and LES are able to compute steady and unsteady flow in multiple hole perforate plates. This has allowed for edge effects due to the partial holes to be shown to have significant influence on the shape and mixing of the merged jet plume.

The individual perofrate jetlets have been shown to be strongly influenced by neighboring jetlets and different flow structures have been observed due to the changes in plate porosity.

The $2^{\text {nd }}$ and $4^{\text {th }}$ order spatio-temporal correlation $R_{i j}$ and $R_{i j, k l}$ confirm acoustic source changes due to the difference in coherent structures. Away from the early jetlet development $2^{\text {nd }}$ and $4^{\text {th }}$ order correlations are similar to those found in an isolated free single jet. The correlations presented here are available as suggested validation data for further acoustic source modeling.

Far-field results confirm that capturing the presence of these flow structures within the acoustic source is important as their influence can be seen in the far-field pressure signal.

\section{Acknowledgment}

The work has been performed under SYMPHONY 'SYstem Manufacturing and Product design tHrough cOmponent Noise technologY', TP11/HVM/6/I/AB201K.

This project was co-funded by the Technology Strategy Board's Collaborative Research and Development programme, following an open competition. The Technology Strategy Board is an executive body established by the Government to drive innovation. It promotes and invests in research, development and the exploitation of science, technology and new ideas for the benefit of business - increasing sustainable economic growth in the UK and improving quality of life.

Computational time on HECToR has been funded by 'Supercomputing Resources and Support for the UK Applied Aerodynamics Consortium 2' EPSRC EP/F005954/1.

Preliminary LES predictions were carried out by Christoph Schäfer as part of his Diploma studies at TU Dresden, supported through ERASMUS. 


\section{References}

${ }^{1}$ Boock, K. and Viertel, M., "Low-Noise Volume Flow Rate Throttling of Fluid-Carrying Pipes," Patent application, AIRBUS DEUTSCHLAND GMBH, July 2007.

${ }^{2}$ Itsuro, H. Teruo, H. and Hiroshi., "Flow Induced Noise at Orifice Plate in Piping Systems," Nippon Kikai Gakkai Kankyo Kogaku Sogo Shinpojiumu Koen Ronbunshu, Vol 14, pp26-29, 2004

${ }^{3}$ Demetz, F. C. Matis, M. F. Langley, R. S. and Wilson, J. L., "Noise radiation from subsonic air flow through single and multiholed orifice plates," Interim Report Naval Ship Research and Development Center, Bethesda, MD. Acoustics Dept., Feb 1979.

${ }^{4}$ Peat, K. S., "A numerical decoupling analysis of perforated pipe silencer elements," Journal of Sound and Vibration, 123(2), 199-212, 1988.

${ }^{5}$ Page, G. J., McGuirk, J. J., Hossain, M., Self, R. H., Bassetti, A., "A CFD Coupled Acoustics Approach for Coaxial Jet Noise," AIAA-2003-3286, 9th AIAA/CEAS Aeroacoustics Conference, Hilton Head, South Carolina, May 2003.

${ }^{6}$ Azarpeyvand, M, Self, R. and Golliard, J., "Improved Jet Noise Modeling Using a New Acoustic Time Scale," AIAA2006-2598, 12th AIAA/CEAS Aeroacoustics Conference, Boston, May 2006.

${ }^{7}$ McMullan, W. A., "Numerical experiments on spatially developing mixing layers using Large Eddy Simulation," $P h . D$. Thesis, University of Leicester, 2005.

${ }^{8}$ Andersson, N., Eriksson, L-E. and Davidson, L., "Effects of inow conditions and subgrid model on LES of turbulent jets," No. 2005-2925, AIAA 2005.

${ }^{9} \mathrm{Wu}, \mathrm{W}$. , Page, G. J., and McGuirk, J. J., "An approcah to improve high frequency noise prediction in LES of jets," 12th AIAA/CEAS Aeroascoutics Coference, Cambridge, Massachusetts No.2006-2442, May 2006,

${ }^{10}$ Freund, J. B., "Noise sources in a low-Reynolds number turbulent jet at Mach 0.9," Journal of Fluid Mechanics. Vol 438, pp 277-305.

${ }^{11}$ Lighthill, M. J., "On sound generated aerodynamically I. General theory," Proc. Roy. Soc. Lond., 1952.

${ }^{12}$ Lighthill, M. J., "On sound generated aerodynamically II. Turbulence as a source of sound," Proc. Roy. Soc. Lond., 1954.

${ }^{13}$ Moinier, P.J., "Algorithm Developments for an Unstructured Viscous Flow Solver," PhD Thesis, University of Oxford, 1998.

${ }^{14}$ Smagorinsky, J., "General circulation experiments with the primitive equations," Monthly Weather Review, Vol. 91, pp 99-164. 1963

${ }^{15}$ Fleury, V., Bailly, C., Juve, D., Michard, M., and Jondeau, E., "Space-time correlations in two subsonic jets using dual-PIV measurements," 13th AIAA/CEAS Aeroacoustic Conference AIAA Paper 2007-3615, 2007.

${ }^{16}$ McMullan, W. A., Pokora, C. D., Page, G. J., and McGuirk, J. J., "Large Eddy Simulation of a High Reynolds Number Subsonic Turbulent Jet for Acoustic Source Capture," 14th AIAA/CEAS Aeroacoustics Conference (29th AIAA Aeroacoustics Conference), AIAA 2008-2974, 5 - 7 May 2008.

${ }^{17}$ Pokora, C. D., and McGuirk, J. J., "Spatio-Temporal Turbulence Correlations using High-Speed PIV in an Axisymmetric Jet," 14th AIAA/CEAS Aeroacoustics Conference (29th AIAA Aeroacoustics Conference), AIAA 2008-3028, 5 - 7 May 2008.

${ }^{18}$ Harper-Bourne, M., "Jet Noise Turbulence Measurements," 9th AIAA/CEAS Aeroacoustic Conference, AIAA Paper 2003-3214, 2003. 